\title{
Underutilised fruits: a review of phytochemistry and biological properties
}

\author{
Ahmad Hasan Salahuddin Mirfat ${ }^{\mathrm{a}, \mathrm{b}^{*}}$, Ismail Amin ${ }^{\mathrm{a}^{*}}, \operatorname{Kassim}_{\text {Nur Kartinee }}^{\mathrm{c}}$, \\ Hamid Muhajird and Mat Ali Mohd Shukri ${ }^{b}$
}

\begin{abstract}
aDepartment of Nutrition and Dietetics, Faculty of Medicine and Health Sciences, University Putra Malaysia, 43400 Serdang, Selangor, Malaysia

${ }^{b}$ Malaysian Agricultural Research and Development Institute, Persiaran MARDI-UPM, 43400 Serdang, Selangor, Malaysia

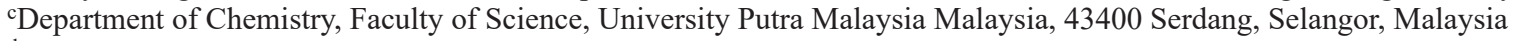

dDepartment of Microbiology, Faculty of Biotechnology and Biomolecular Sciences, University Putra Malaysia, 43400 Serdang, Selangor, Malaysia

*Corresponding author: Ahmad Hasan Salahuddin Mirfat, Ismail Amin, Department of Nutrition and Dietetics, Faculty of Medicine and Health Sciences, University Putra Malaysia, 43400 Serdang, Selangor, Malaysia. Tel: +603-89472435; Fax: +603-89426769;

E-mail: mirfat@mardi.gov.my, aminis@upm.edu.my
\end{abstract}

DOI: $10.31665 /$ JFB.2018.1124

Received: February 26, 2018; Revised received \& accepted: March 27, 2018

Citation: Mirfat, A.H.S., Amin, I., Nur Kartinee, K., Muhajir, H., and Mohd Shukri, M.A. (2018). Underutilised fruits: a review of phytochemistry and biological properties. J. Food Bioact. 1: 2-30.

\begin{abstract}
Underutilised fruits are not only important sources of food and nutrition, but also secure household income especially for rural and farm communities. However, some of the underutilised fruits have not received much attention as compared to commercial fruits. This could be due to their lack of knowledge of their potential values. Hence, information about their health promoting properties is critical to increase the value of underutilised fruit species to enhance their preservation and sustainable use in strengthening food, nutrition, health and livelihood security. This article aims to provide a comprehensive review on the phytochemical properties and biological activities of underutilised fruit species grown in Malaysia focussing on health promoting aspects. With regard to phytochemistry, only 21 species of underutilised fruits have been identified and quantified. Phytochemical investigations of various parts of the fruits have revealed the presence of over 100 phytochemicals which are phenolics, terpenoids, carotenoids and other miscellaneous compounds. About 51 underutilised fruit species have been explored for interesting biological activities (antioxidant, antimicrobial, anticholinesterase, cytotoxicity, antiatherosclerotic, antihyperlipidemia, antidiabetic, cytoprotective, cardioprotective and antiplatelet activities) supporting their diverse traditional uses. Different parts of the fruits have been analysed mainly in vitro and barely in vivo, with pulp being the most dominant. Of all the underutilised fruits studied, Mangifera species and Canarium odontophyllum have been the major focus for researchers. The gaps obtained from this review create further research opportunities to add to the current knowledge of health promoting properties of underutilised fruits in Malaysia. More studies are needed to confirm the health significance and explain their mechanisms of action in order to fully understand the real potential of this underutilised fruit species.
\end{abstract}

Keywords: Underutilised fruits; Traditional uses; Phytochemical compounds; Biological activities. 


\section{Introduction}

Malaysia possesses a rich diversity of underutilised fruits grown in orchards, home gardens and some can be found in the wild of Peninsular Malaysia, Sabah and Sarawak, which are located on the Borneo Island. These underutilised fruits are important sources of food and nutrition for rural and farm communities, which improve the quality of diets and sustenance of the communities. The diversity of the fruit species in the fruit orchards or home gardens is not only an important source of food and nutrition, but also probably more importantly secure household income and thus leads to the improvement of their livelihood (Salma et al., 2006). Other than being popularly consumed as a source of food, some of the underutilised fruits have also been used in folk medicine. The comprehensive list of the ethnobotanical uses of over 50 Malaysian underutilised fruits from 23 families is displayed in Table 1. This information can be the basis for further research to investigate the health and phytochemical aspects of underutilised fruit species. Anacardaceae family which consists of one species of Bouea macrophylla and seven species of the genus Mangifera, is the most studied underutilised fruit in Malaysia. Anacardaceae fruits are mainly used as food source and only some have been reported to possess medicinal properties to treat various ailments such as $M$. caesia, M. foetida, M. pajang and M. pentandra (Gerten et al., 2015; Khoo et al., 2016; Mirfat et al., 2016; Mirfat et al., 2015; Salma et al., 2006). Euphorbiaceae, Guttiferae and Sapindaceae are the next commonly studied underutilised fruits in Malaysia. However, there are no reports in the literature on the ethnomedicinal properties of Euphorbiaceae (Baccaurea angulata, B. lanceolata, $B$. macrocarpa, B. motleyana and B. polyneura) other than being a source of food. Nephelium ramboutan-ake from the Sapindaceae family has been described to treat scabbies and itchiness (Gerten et al., 2015). Guttiferae which are usually used in cooking, have been reported to have medicinal potential as shown by Garcinia atroviridis (Gerten et al., 2015; Salma et al., 2006), G. dulcis (Abu Bakar et al., 2015) and G. parvifolia (Gerten et al., 2015; Salma et al., 2006). In addition, other underutilised fruits species such as Parkia speciosa and Averrhoa bilimbi have also been widely reported by researchers to have various traditional remedial use. Parkia speciosa has been used for its diuretic and relaxing properties, treatment of high blood pressure, diabetes and has antibacterial effects on kidney, ureter and urinary bladder (Ko et al., 2014; Salma et al., 2006; Voon and Kueh, 1999) . Meanwhile, A. bilimbi is useful in treating fever, cold, coughs, itches, boils, beriberi, biliousness, inflammation of the rectum, internal haemorrhoids, hypertension, diabetes, syphilis and rheumatism (Khoo et al., 2016; Muhamad et al., 2014; Noor \& Noriham, 2014; Salma et al., 2006).

However, as these underutilised fruits are usually maintained by cultural preferences and traditional practices, some of them have been largely neglected in research and conservation. They have also not received much attention as compared to commercial fruits. This could be due to their lack of knowledge of their potential value and also promotional campaigns. Information about their health promoting properties is critical to increase the value of such neglected species to enhance the preservation and sustainable use of these underutilised fruits in strengthening food, nutrition, health and livelihood security. Therefore, this review was conducted to describe the current progress in research regarding the phytochemical properties and biological activities of underutilised fruit species in Malaysia which cover various health promoting aspects. The gaps present will further highlight existing research opportunities to enhance the current knowledge of health promoting properties of underutilised fruits in Malaysia.

\section{Phytochemistry}

Phytochemicals are non-nutritive bioactive compounds naturally produced by plants that can be categorized into phenolics, alkaloids, terpenes and steroids, among others (Chew et al., 2011). Table 2 summarizes the major phytochemical components of 21 species of Malaysian underutilised fruits. Over 100 phytochemicals were identified and isolated from different parts of the fruits with pulp being the mostly studied. Phenolics have been identified as the most vital phytochemical compounds that contribute to the diverse health properties reported. Of the phenolic compounds, the most important reported were gallic acid, catechin and protocatechuic acid (Abu Bakar et al., 2010; Ahmad et al., 2015; Ahmed et al., 2013; Hassan et al., 2011; Khoo et al., 2012a; Prasad et al., 2011a; 2011b; Sulaiman and Ooi, 2012; Tee et al., 2015). Among the most useful and powerful analytical instruments used to investigate these compounds are high performance liquid chromatography (HPLC), gas chromatography-mass spectrometry (GC-MS) and liquid chromatography-mass spectrometry (LC-MS).

The Anarcardiaceae family which consists of $M$. caesia, $M$. foetida, M. odorata, $M$. pajang, $M$. pentandra and $M$. quadrifida dominates the number of the phytochemical studies. Gallic acid, vanillic acid and mangiferin were among the compounds found especially in the pulp of the fruit species (Sulaiman and Ooi, 2012). However, M. pajang fruit parts were thoroughly investigated by researchers. The peel was reported to contain various classes of phenolics including pyrogallic acid, gallic acid, catechin, epicatechin, mangiferin, rutin, protocatechuic acid, chlorogenic acid, methyl gallate, 4-hydroxybenzoic acid, vanillic acid, ethyl gallate, p-coumaric acid, ferulic acid, ellagic acid, morin, daidzein, kaempferol, luteolin, diosmin, quercetin, naringin, hesperidin, caffeic acid, chlorogenic acid and sinapic acid (Abu Bakar et al., 2010; Ahmad et al., 2015; Hassan et al., 2011; Prasad et al., 2011b).

A broad phytochemical study was also conducted in Canarium odontophyllum or fondly known as dabai. All the fruit parts comprising pulp, peel, pulp with peel, pericarp and seed were investigated. The chemical composition differs largely according to the fruit parts. Catechin, epicatechin, epigallocatechin, epicatechin gallate, methyl gallate, ellagic acid and vanillic acid were the major phenolics reported in the pulp and peel (Khoo et al., 2012a). Another study by Khoo et al. (2013) indicated that the peel and pericarp contained cyanidin-3-glucoside, cyanidin-3-galactoside and cyanidin-3-arabinoside. These anthocyanins are one of the many classes of phenolics that are normally found in fruits. Meanwhile, all -trans- $\beta$-carotene, 13 -cis- $\beta$-carotene, all-trans-lutein, 9 -cis-lutein, 13 -cis-lutein, di-cis- $\beta$-carotene, 15 -cis- $\beta$-carotene and 9 -cis- $\beta$-carotene were the carotenoids present in almost all parts of the fruits (Prasad et al., 2011a)

A phytochemical screening of various fruit parts of Litsea garciae, locally known as engkala, has resulted in the identification of viniferin, cyanidin, ferulic acid (pulp), dihydroquercetin, $p$ coumaroyl, tartaric acid, caffeoyl tartaric acid, cinnamoyl glucose (seed), ferulic acid, cinnamoyl glucose, epigallocatechin (cupule) and viniferin, elphinidin 3,5-O-diglucoside, cinnamoyl glucose (peel) (Husen, 2015). Baccaurea angulata peel, pulp and whole fruit were phytochemically investigated by Ahmed et al. (2013; 2015) in two different studies. Both studies confirmed the presence of catechin, ascorbic acid, vanillic acid, carnosic acid, cinnamic acid, caffeic acid and myricetin in the different parts of the fruit (Ahmed et al., 2013; Ahmed et al., 2015). The most recent study of the Malaysian underutilised fruits was reported by Abu Bakar et al. (2016). They analysed the chemical constituents of Rubus moluccanus, $R$. fraxinifolius and $R$. alpestris using a GC-MS. The results 


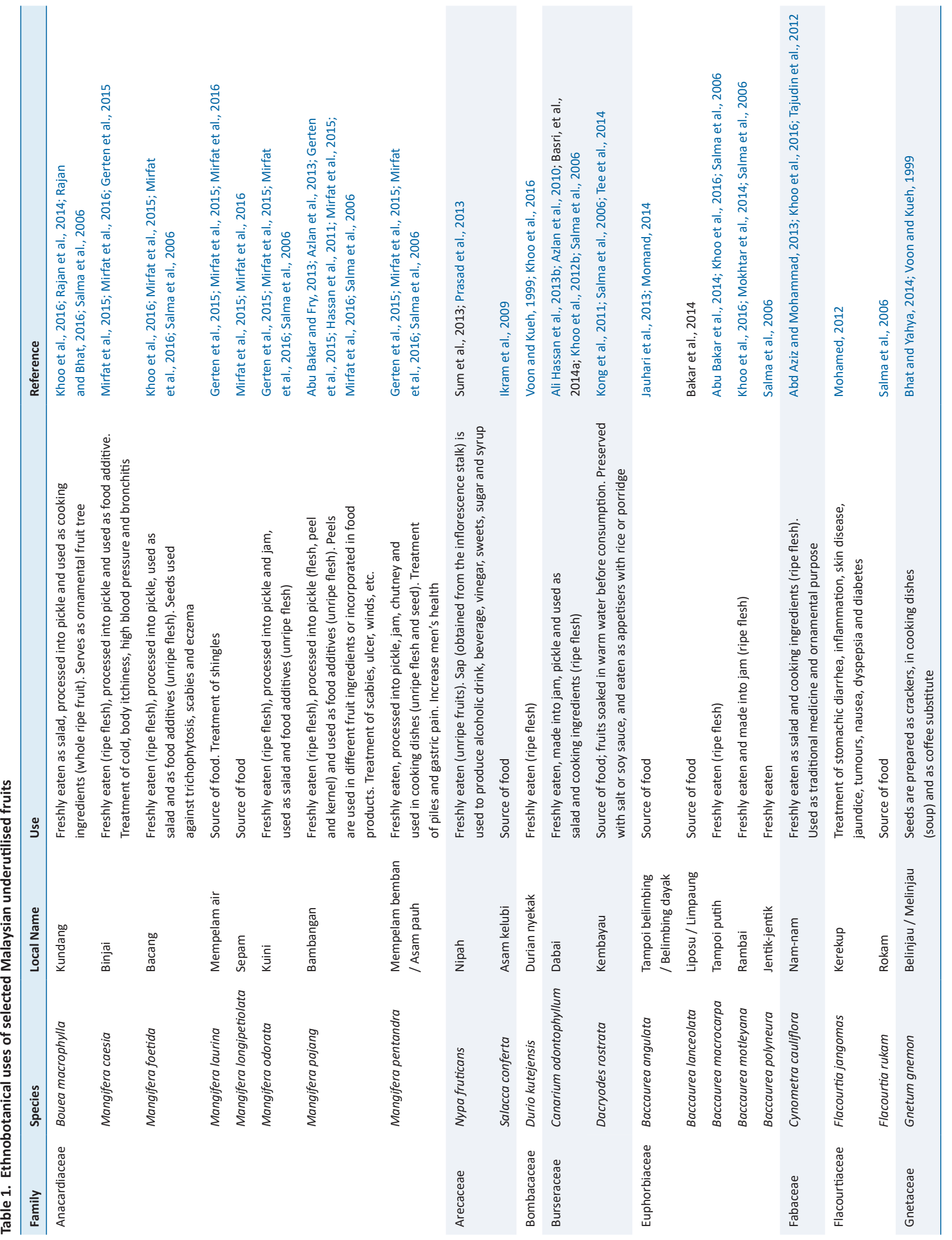




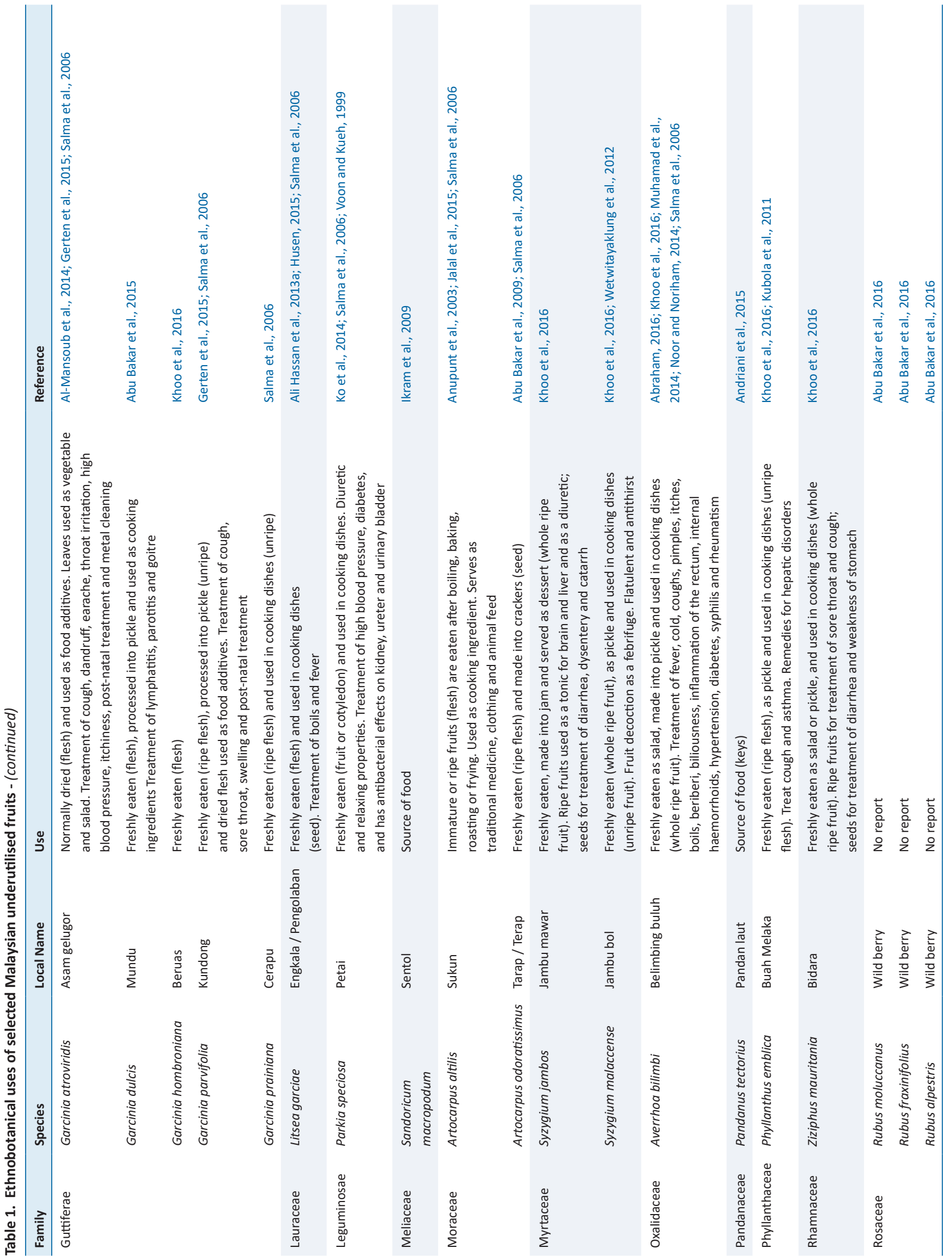




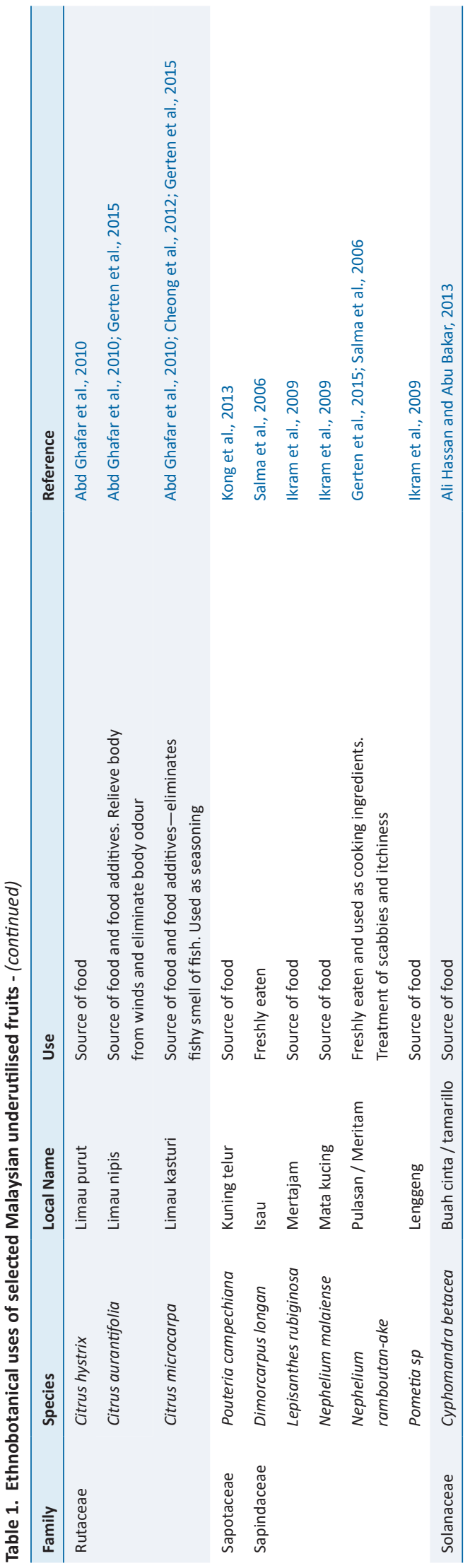

revealed the presence of at least 12,21, and 7 different compounds in $R$. alpestris, $R$. moluccanus and $R$. fraxinifolius, respectively.

\section{Biological activities}

Based on the literature search, the health promoting aspects of Malaysian underutilised fruits have not been thoroughly investigated, as shown in Tables 2, 3 and 4. Antioxidant effect has dominated the activities $(63 \%)$ as it is the basis study to lead to possibilities of finding other therapeutic potentials. A few studies have been reported on antimicrobial activities (14\%) of selected underutilised fruit species. Other biological activities that were documented from Malaysian underutilised fruits include anti-cholinesterase $(9 \%)$, cytotoxicity $(7 \%)$, cytoprotective $(4 \%)$, anti-atherosclerotic (3\%), anti-hyperlipidemia (1\%), antidiabetic $(1 \%)$, cardioprotective $(1 \%)$ and anti-platelete $(1 \%)$. Both in vitro and in vivo assays have been performed to evaluate antioxidant potential, while in vitro assays were used for antimicrobial, anticholinesterase and anticancer activities. Other in vivo experiments were carried out to assess antiatherosclerotic, antihyperlipidemia, antidiabetic, cytoprotective and cardioprotective effects. Pulp was the commonly used fruit part (47\%) in the analyses, followed by peel $(16 \%)$, whole fruit (11\%), seed (9\%) and others. A summary of the findings is presented below.

\subsection{Antioxidant effect}

All underutilised fruit species listed in were tested for their antioxidant capacity. The findings that have been documented showed that different fruit species exhibited varying degrees of antioxidant activities depending on the fruit parts, assays and extraction solvents used (Table 3). With regard to in vitro assays, 2,2-diphenyl1-picrylhydrazyl (DPPH) radical scavenging was the most widely applied. Ferric reducing antioxidant power (FRAP) ranked second, followed by 2-2'-azino-bis (3-ethylbenz-thiazoline-6-sulfonic acid (ABTS) and $\beta$-carotene bleaching activity. The in vitro activities were also measured using metal chelating, Trolox equivalent antioxidant capacity (TEAC), phosphatidylcholine peroxidation, phosphomolybdenum, oxygen radical absorbance capacity (ORAC), copper (II) reduction antioxidant capacity (CUPRAC), thiocyanate, superoxide radical, linoleic acid peroxidation and haemoglobin oxidation assays. With respect to extraction solvents, methanol was the most popular one followed by water and ethanol. Other solvents used were hexane, ethyl acetate, acetone, petroleum ether, chloroform, dichloromethane and phosphate buffered saline (PBS). In vivo assays were conducted using enzymatic and nonenzymatic antioxidant assays in both animals and humans.

Out of 51 underutilised fruit species tested for antioxidant, $C$. odontophyllum demonstrated the highest numbers of antioxidant studies. Various parts of the fruits were analysed almost by the same group of researchers which comprised pulp (Ali Hassan et al.; 2013b; Prasad et al., 2010; Shakirin et al., 2010), pulp with peel (Ali Hassan et al., 2013b; Khoo et al., 2012a; 2012b; Shakirin et al., 2010), peel (Khoo et al., 2012; 2013; 2014; Prasad et al., 2010; Shakirin et al., 2010), seed (Ali Hassan, 2013b; Khoo et al., 2012; Prasad et al., 2010), pericarp (Khoo et al., 2013; 2014), kernel (Shakirin et al., 2010) and whole fruit (Chew et al., 2011). Khoo et al. (2013) and Shakirin et al. (2010) reported that peel extracts showed the highest inhibition percentage as measured by DPPH radical scavenging activity with $60 \%$ and $78.2 \pm 0.5 \%$, respectively. Meanwhile, Prasad et al. (2010) reported that peel exhibited stronger ABTS radical scavenging activity $(84.5 \pm 0.9 \%)$ and higher $\beta$-carotene 


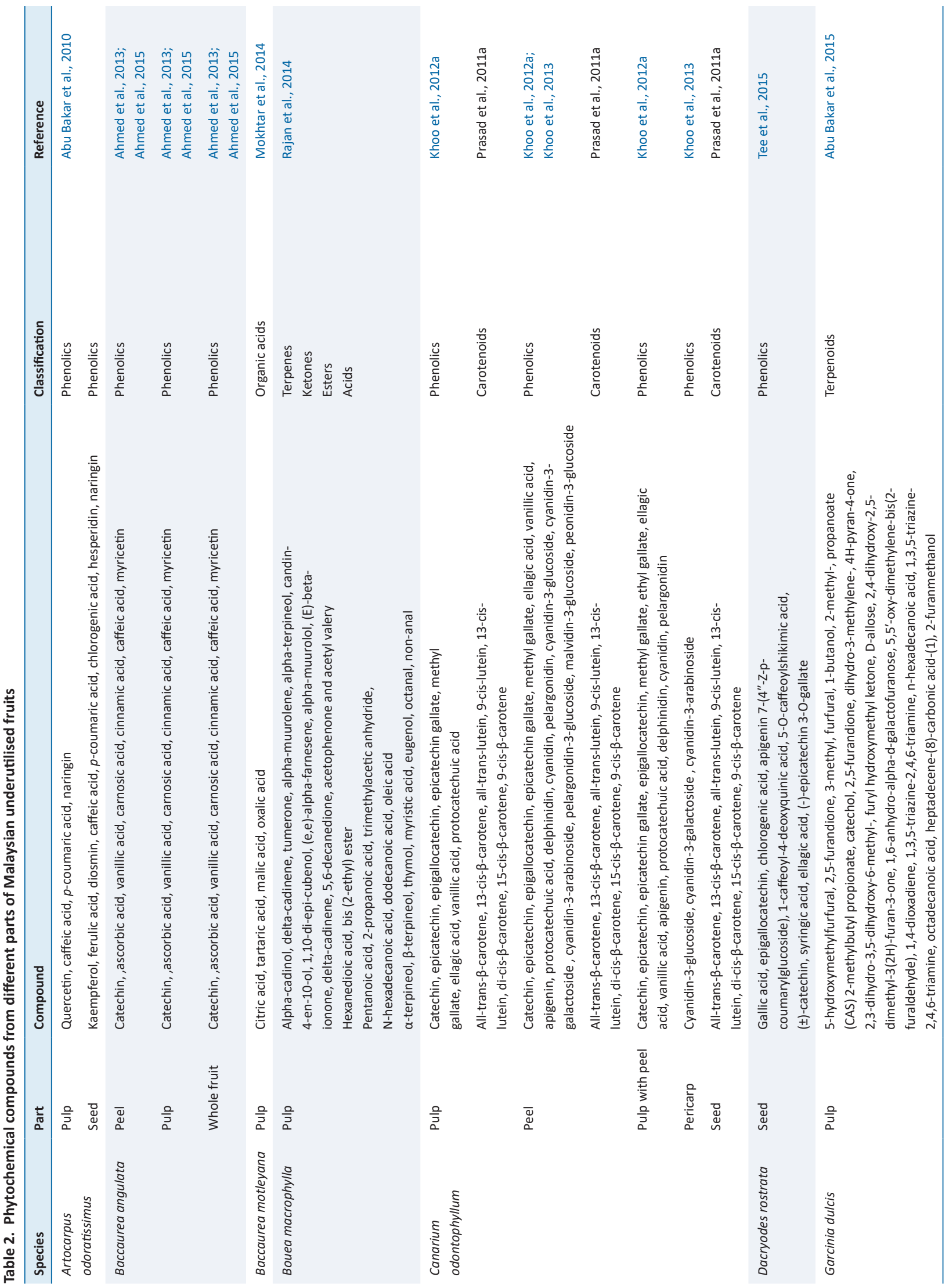




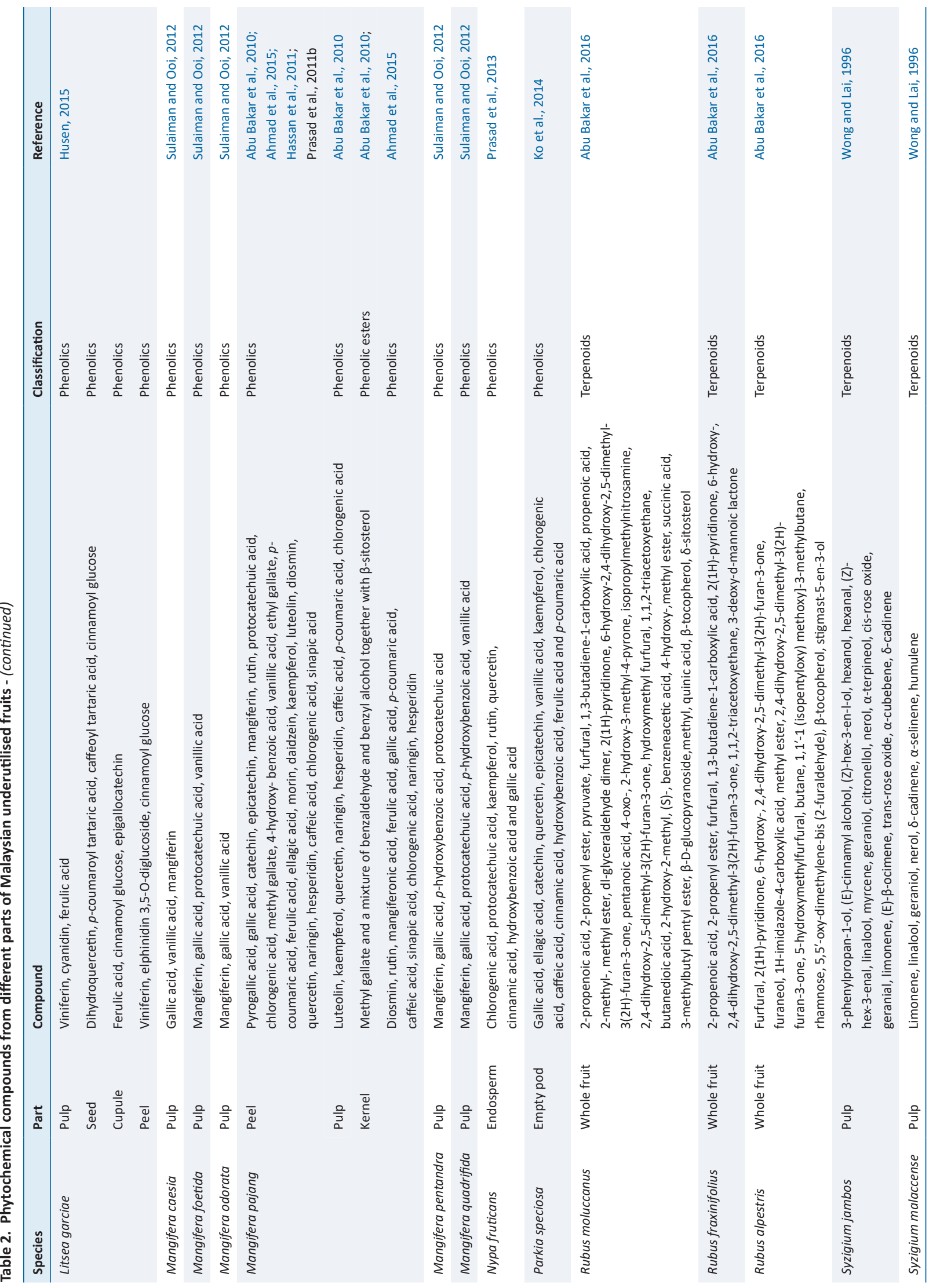




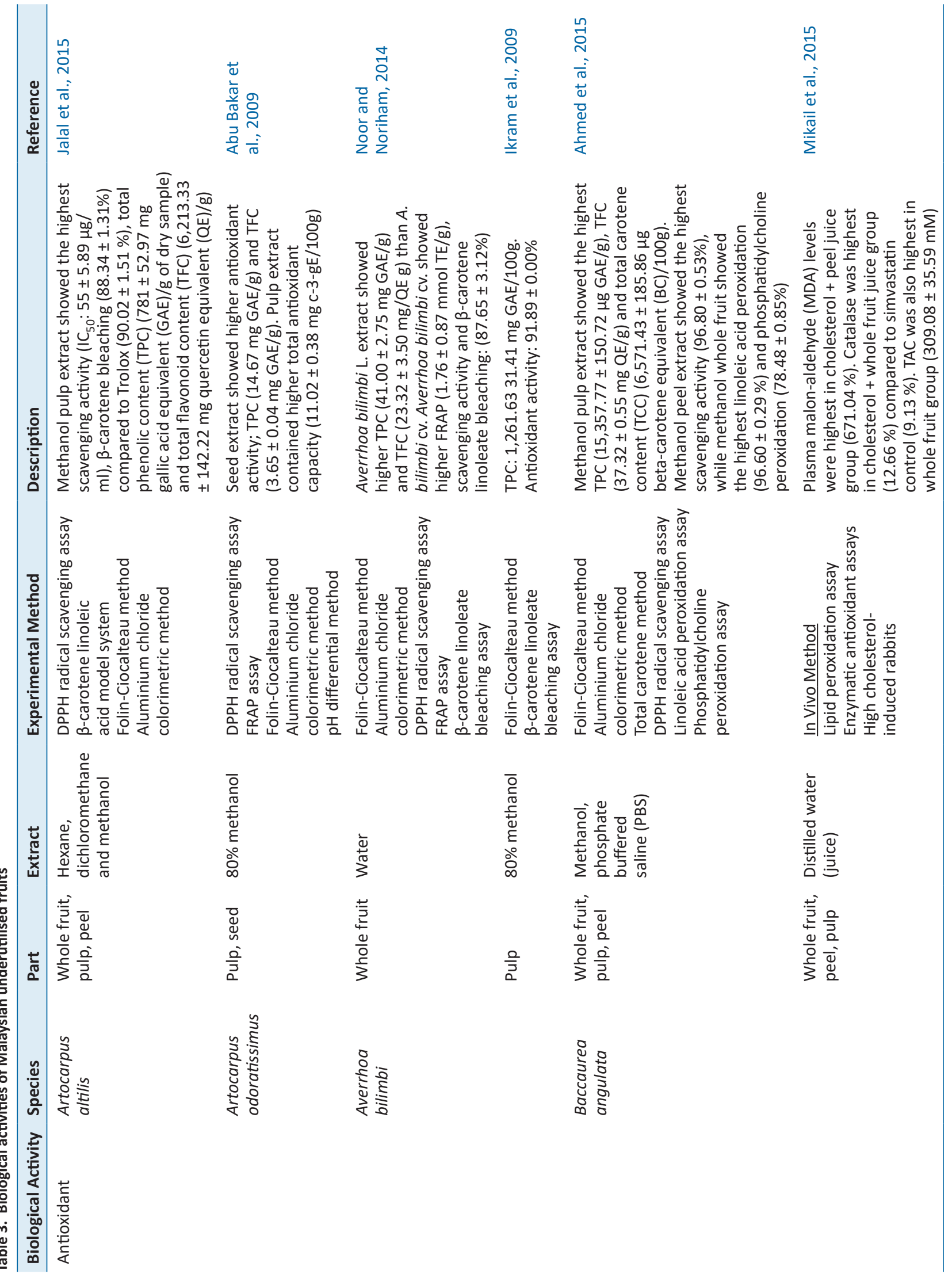




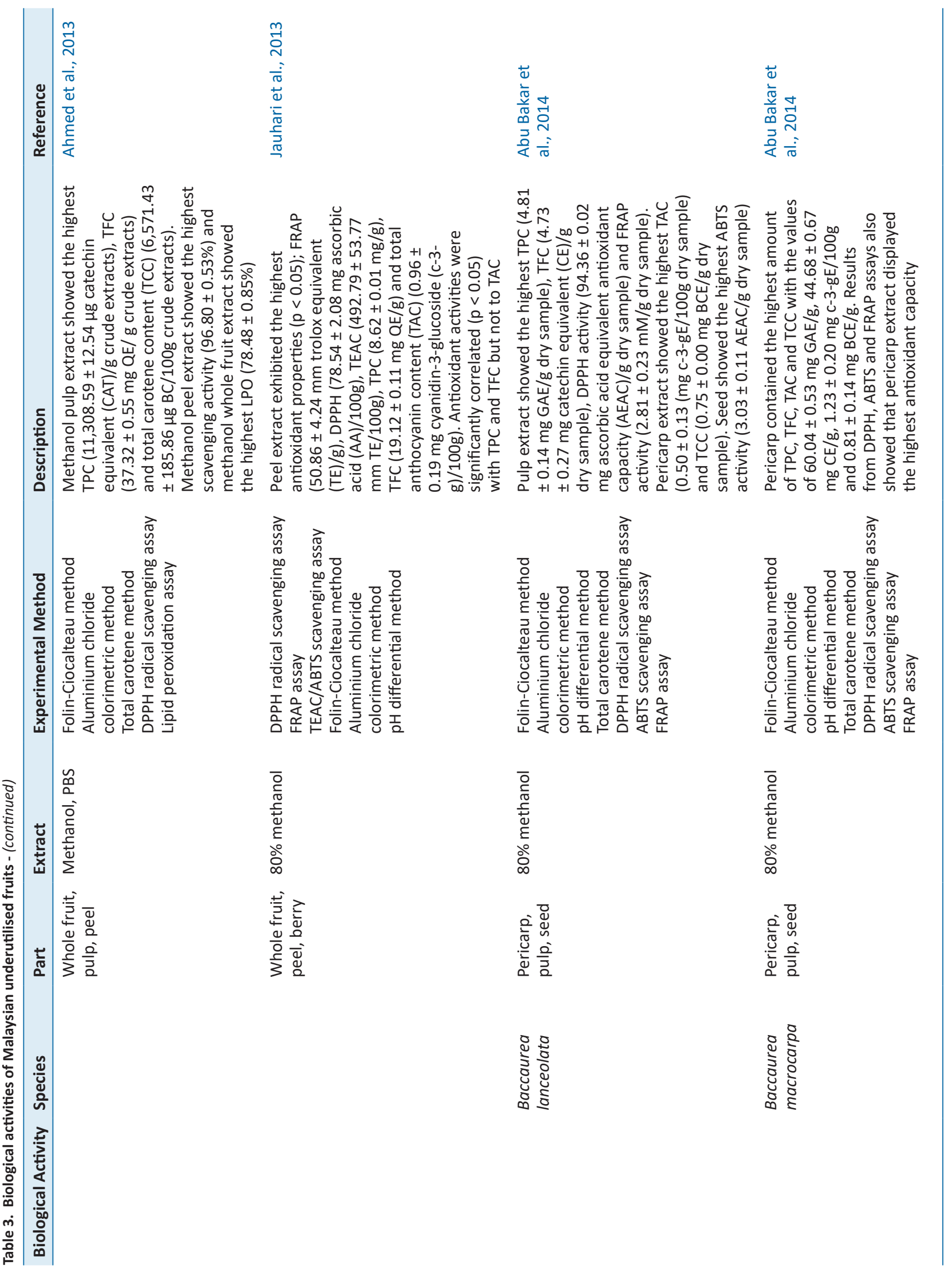




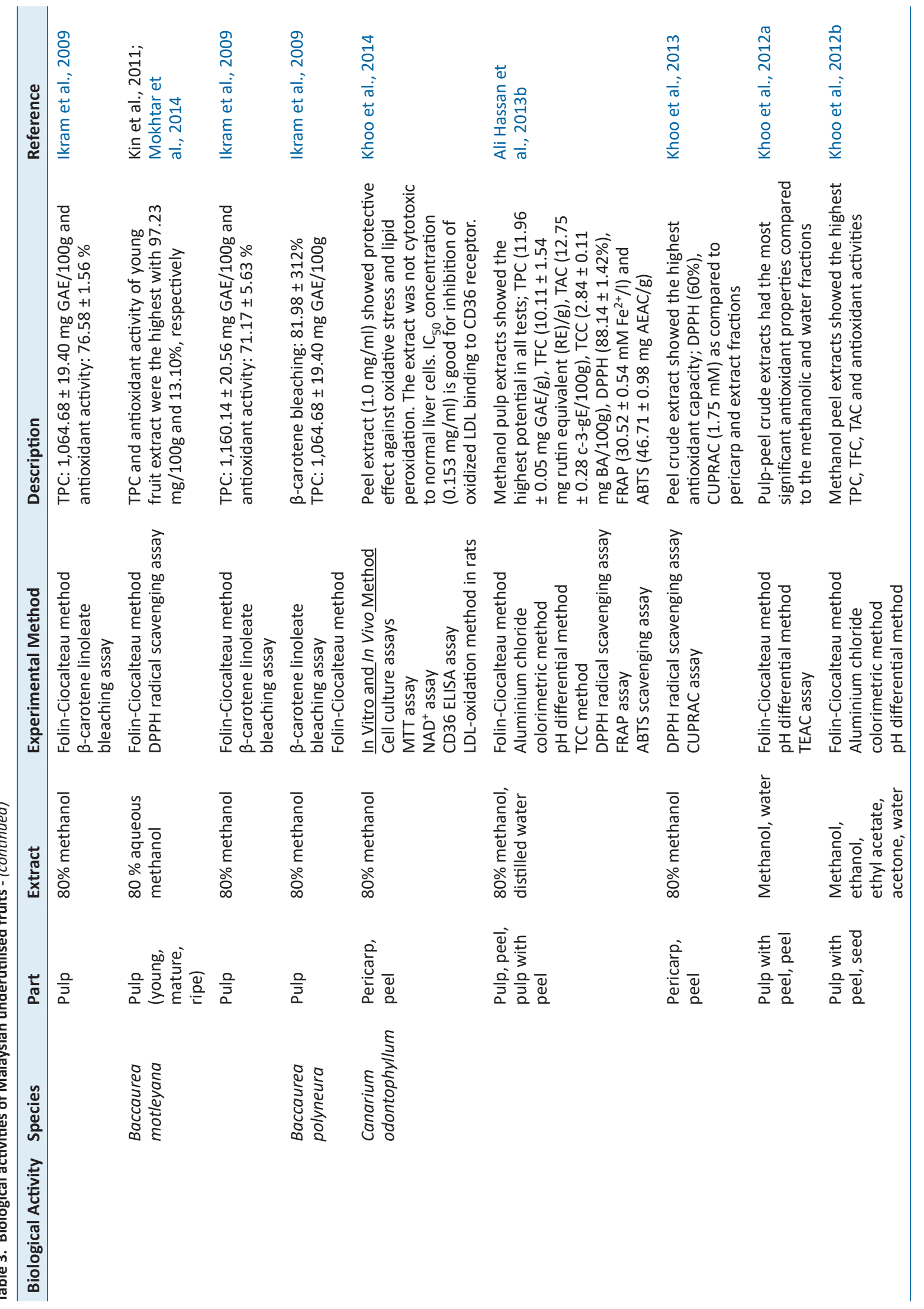




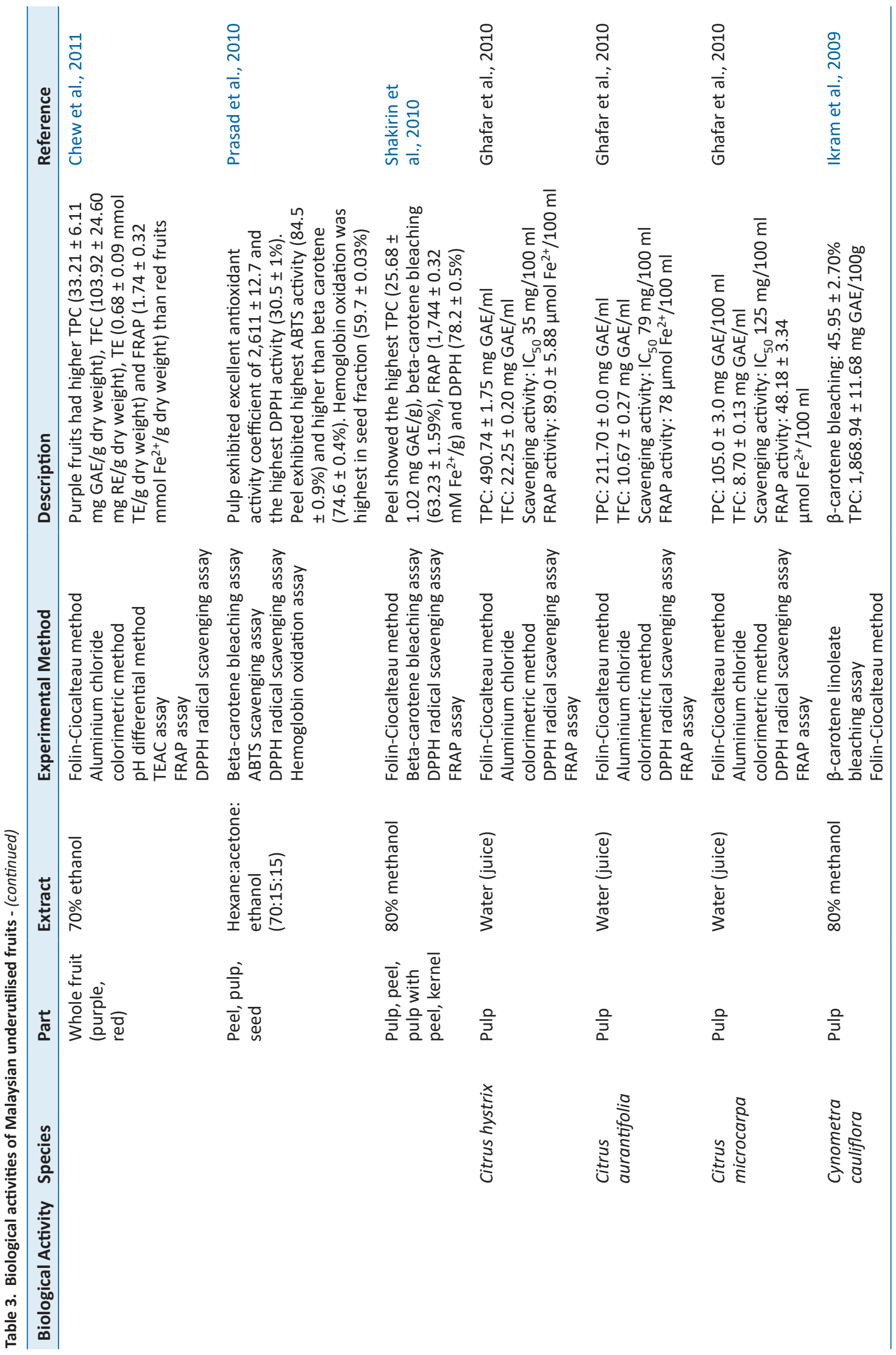




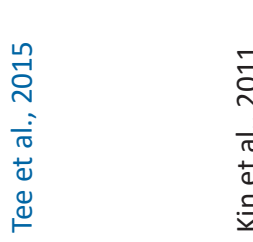

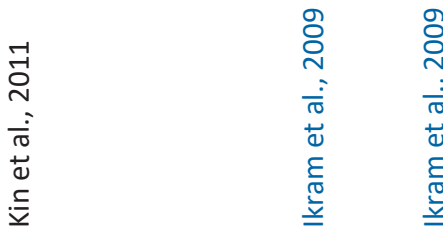

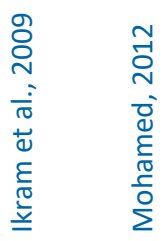

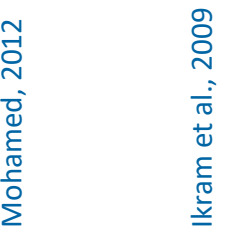

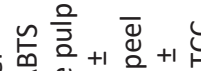

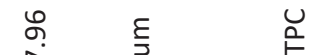

造

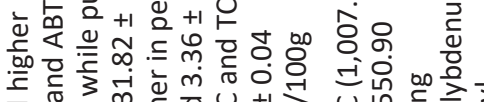

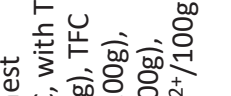

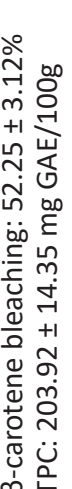

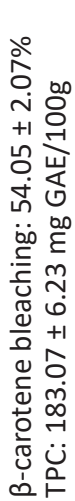

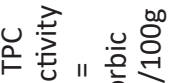

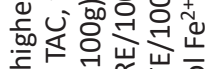

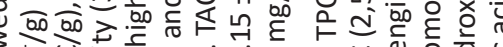

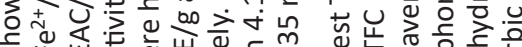

는

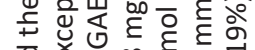

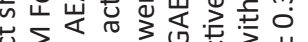

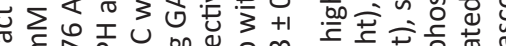

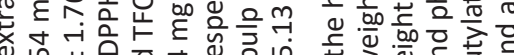

ए

:

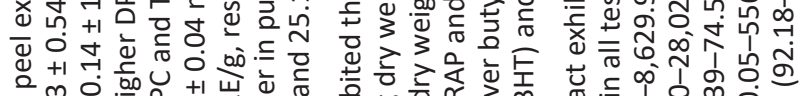

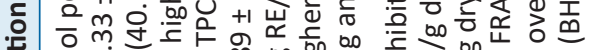

Tू.

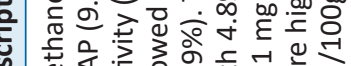

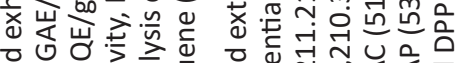

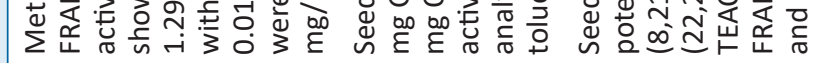

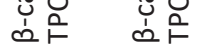

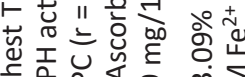

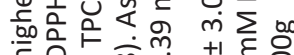

v

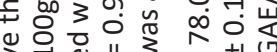

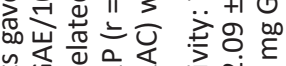

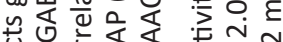

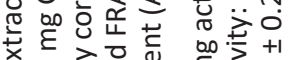

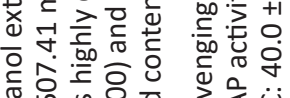

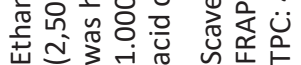

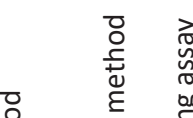

స్

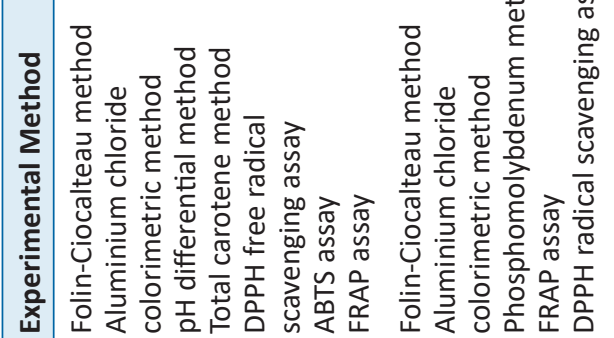

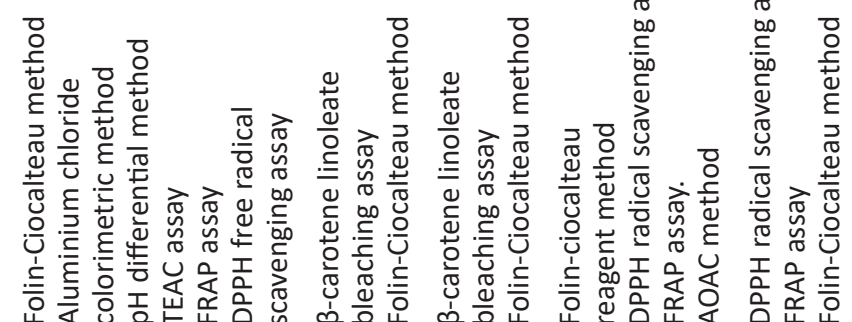

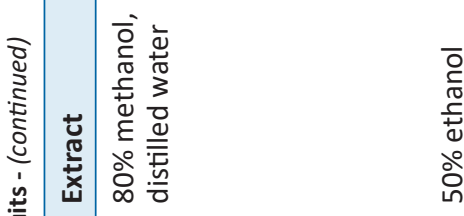

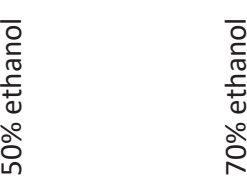

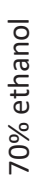

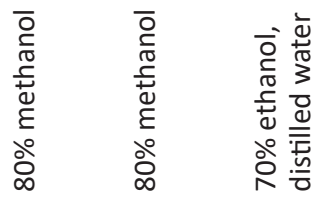

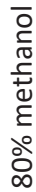

बूँ

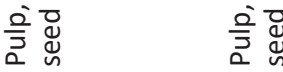

을 음 음

$\frac{0}{3}$

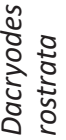

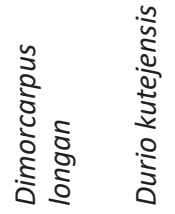

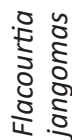

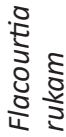




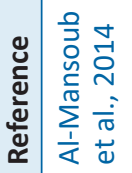

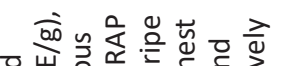

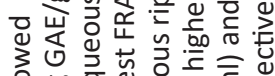

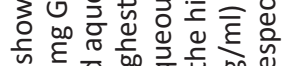

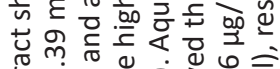

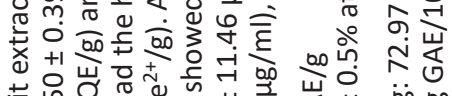

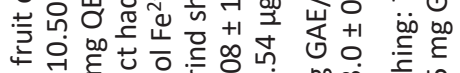

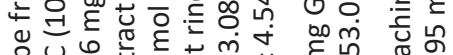

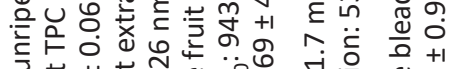

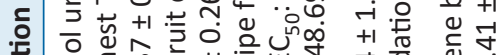

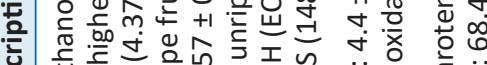

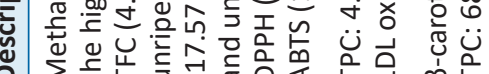

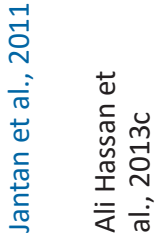

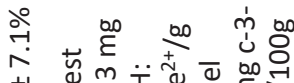

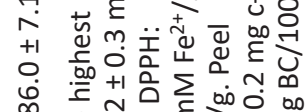

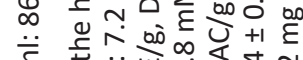
ह

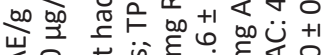
崖它论 no

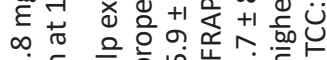
文

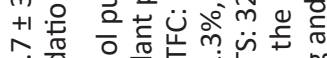

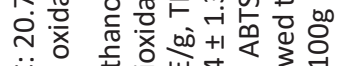

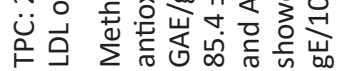

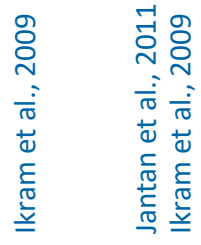

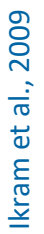

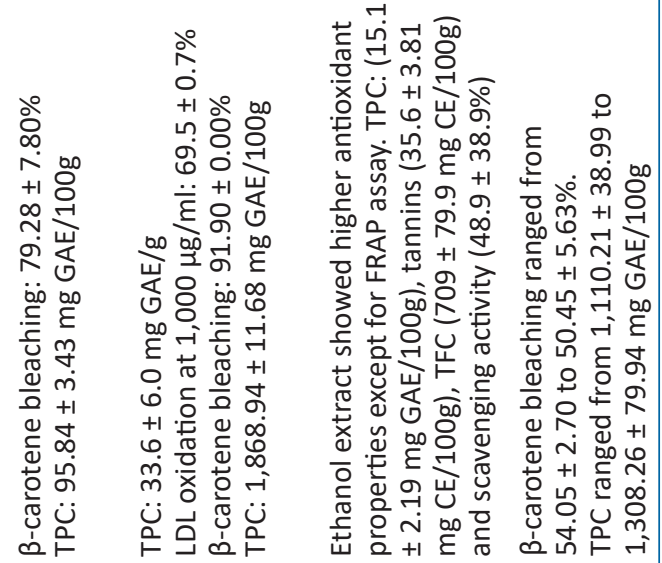

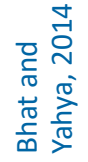

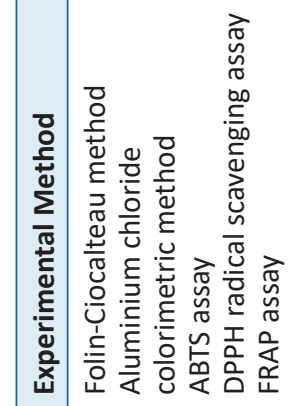

$x^{2}$

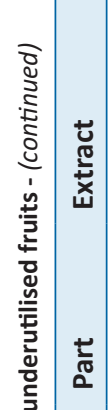

ষ্

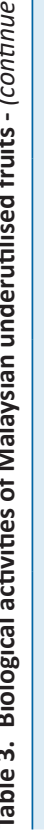

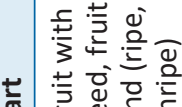

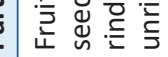

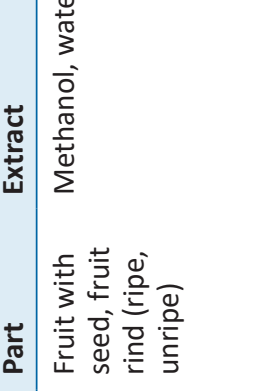

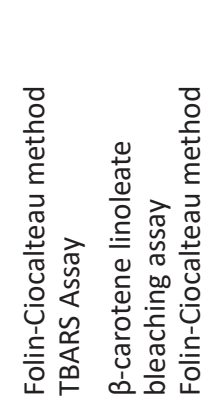
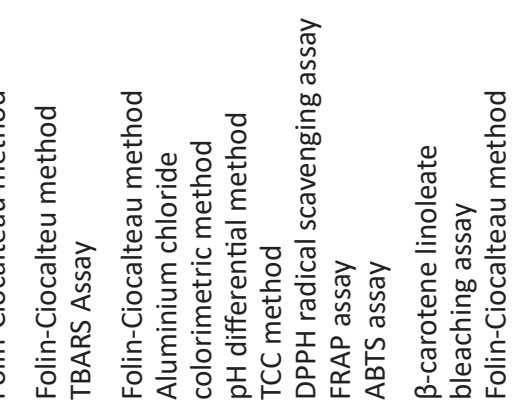

et al.

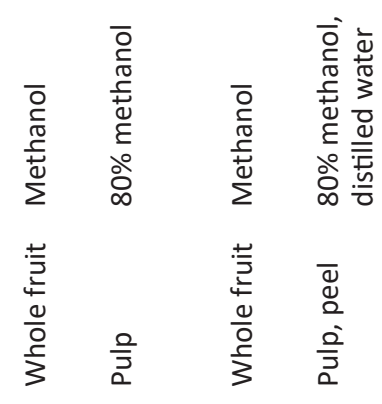

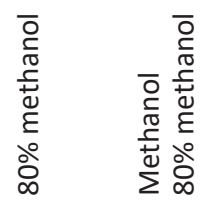

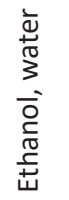

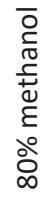

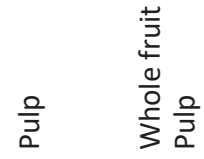

$\frac{0}{3}$

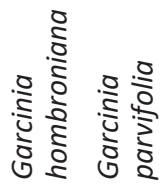

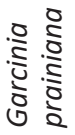

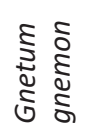

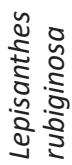




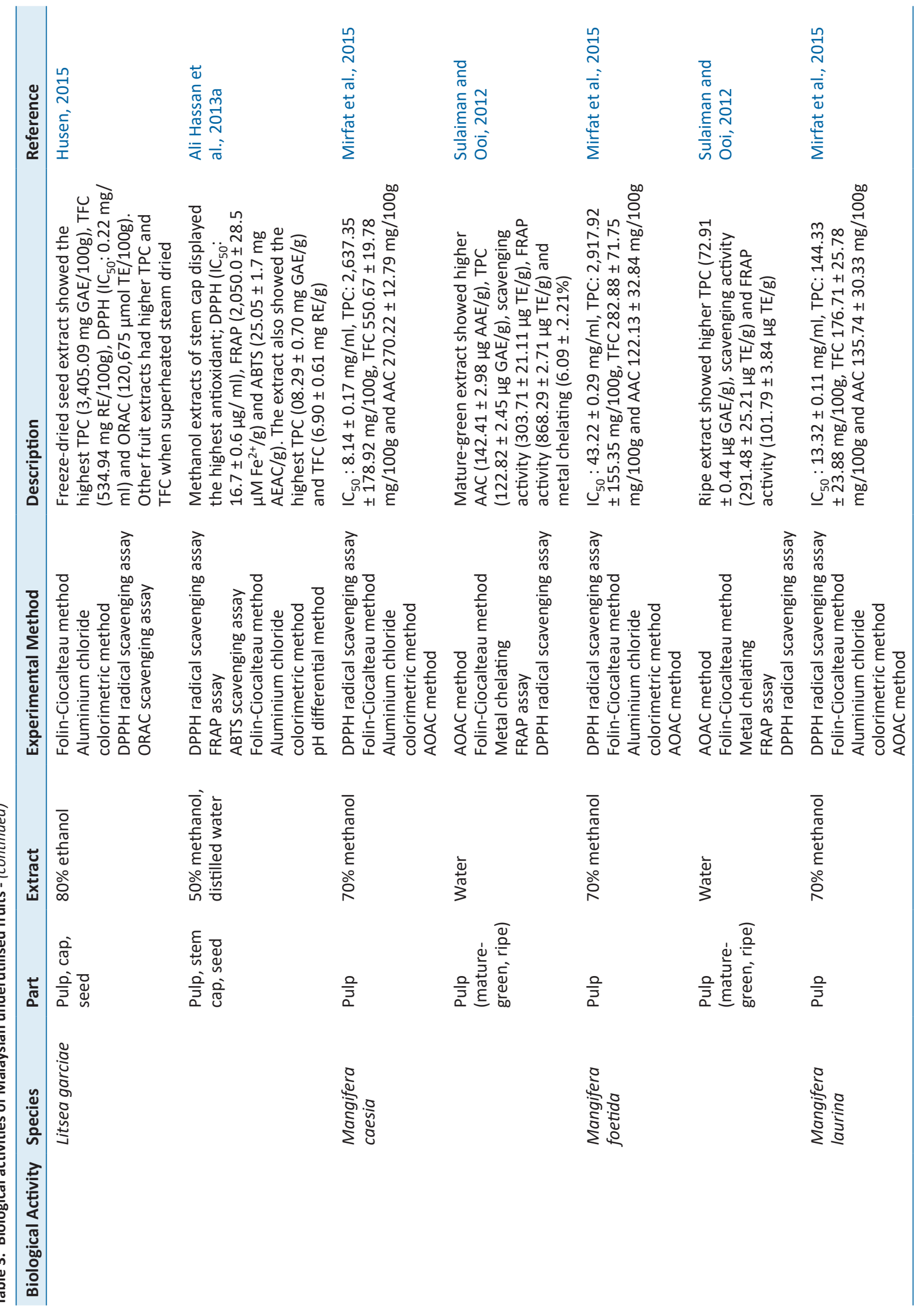




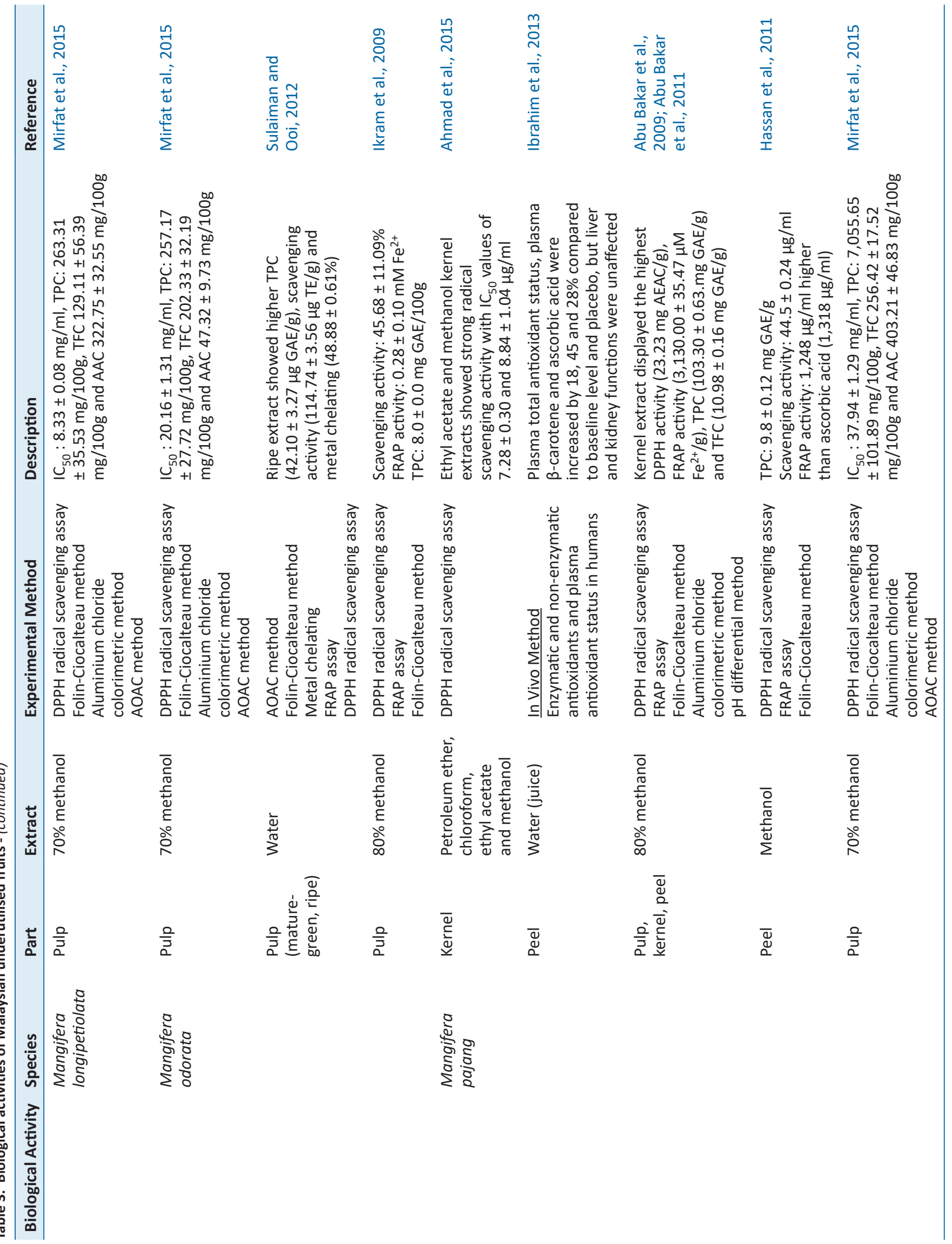




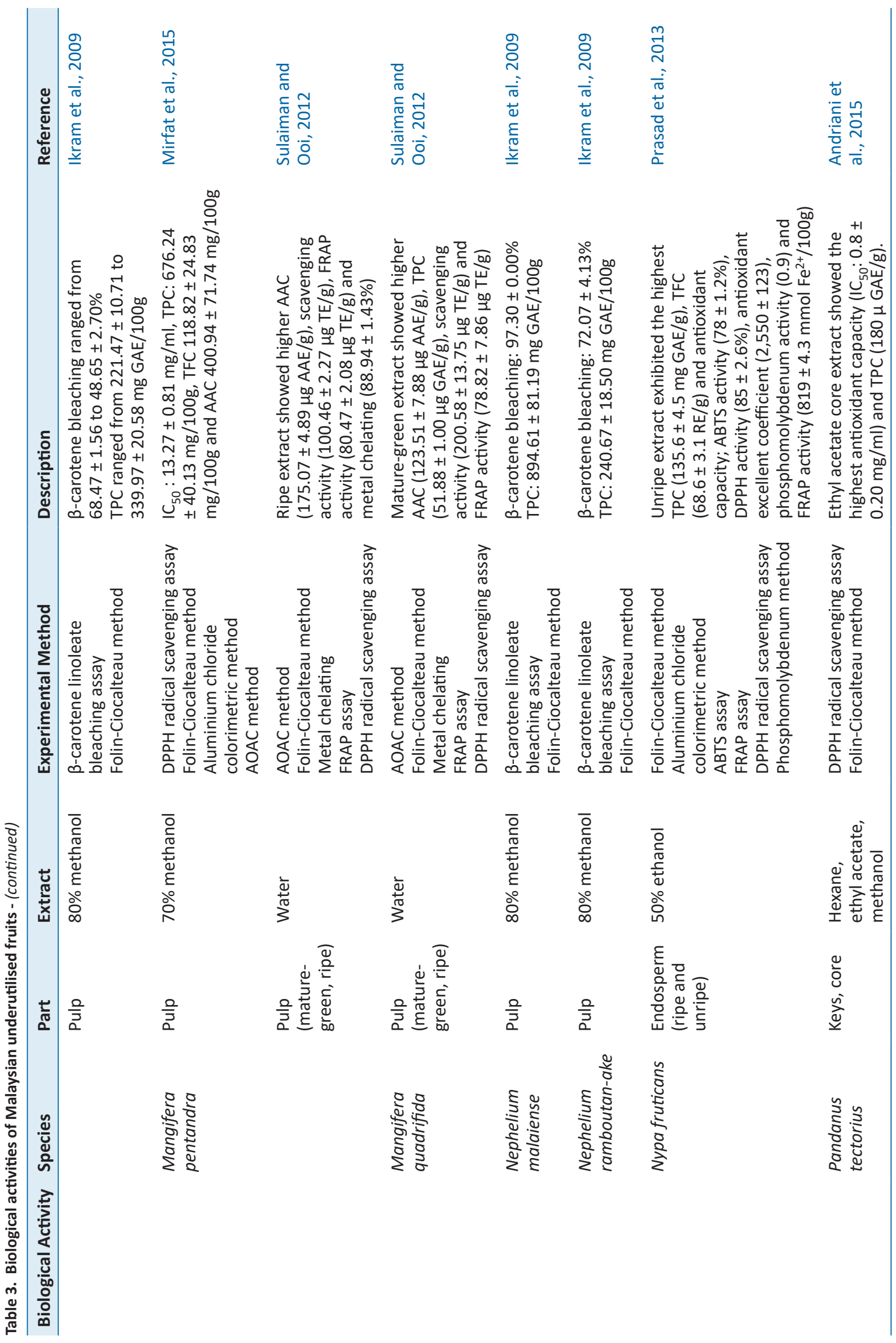




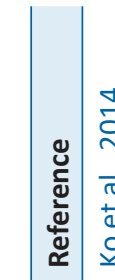

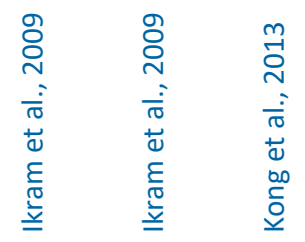

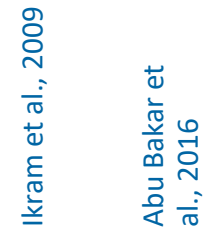

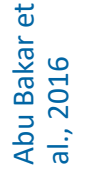

พั่

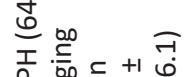

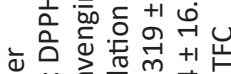

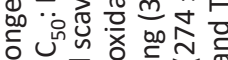

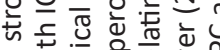

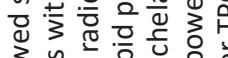

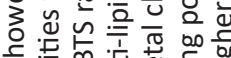
兄: 龸 宕

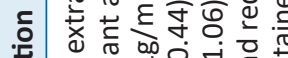

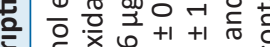

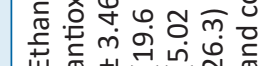

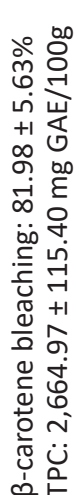

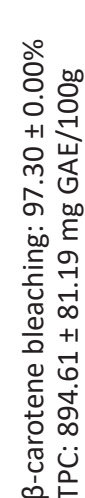

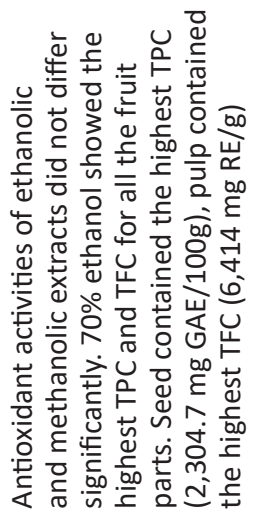

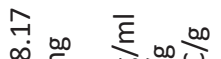

ํํำ نं จे

$\left.\circ \sum \infty 0\right)$ एمٌ

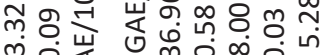

$m \circ$ \& $000+1$

$\ddot{z}+1$ b

>

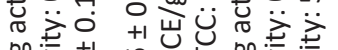

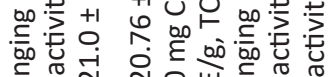

Ð

忢迹占

นึ่ نㅊํㄴ원 म0े யొ 志永 हैنั

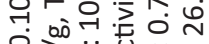
+1 岁苍荠 or 00 o 군듀

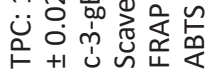

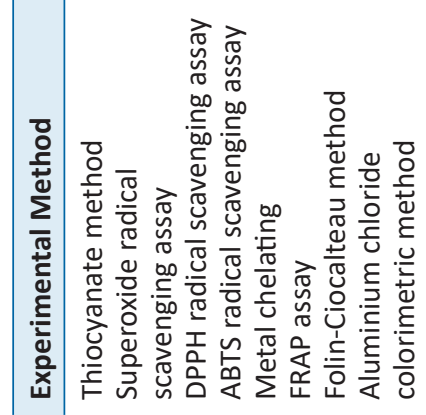

क्षे
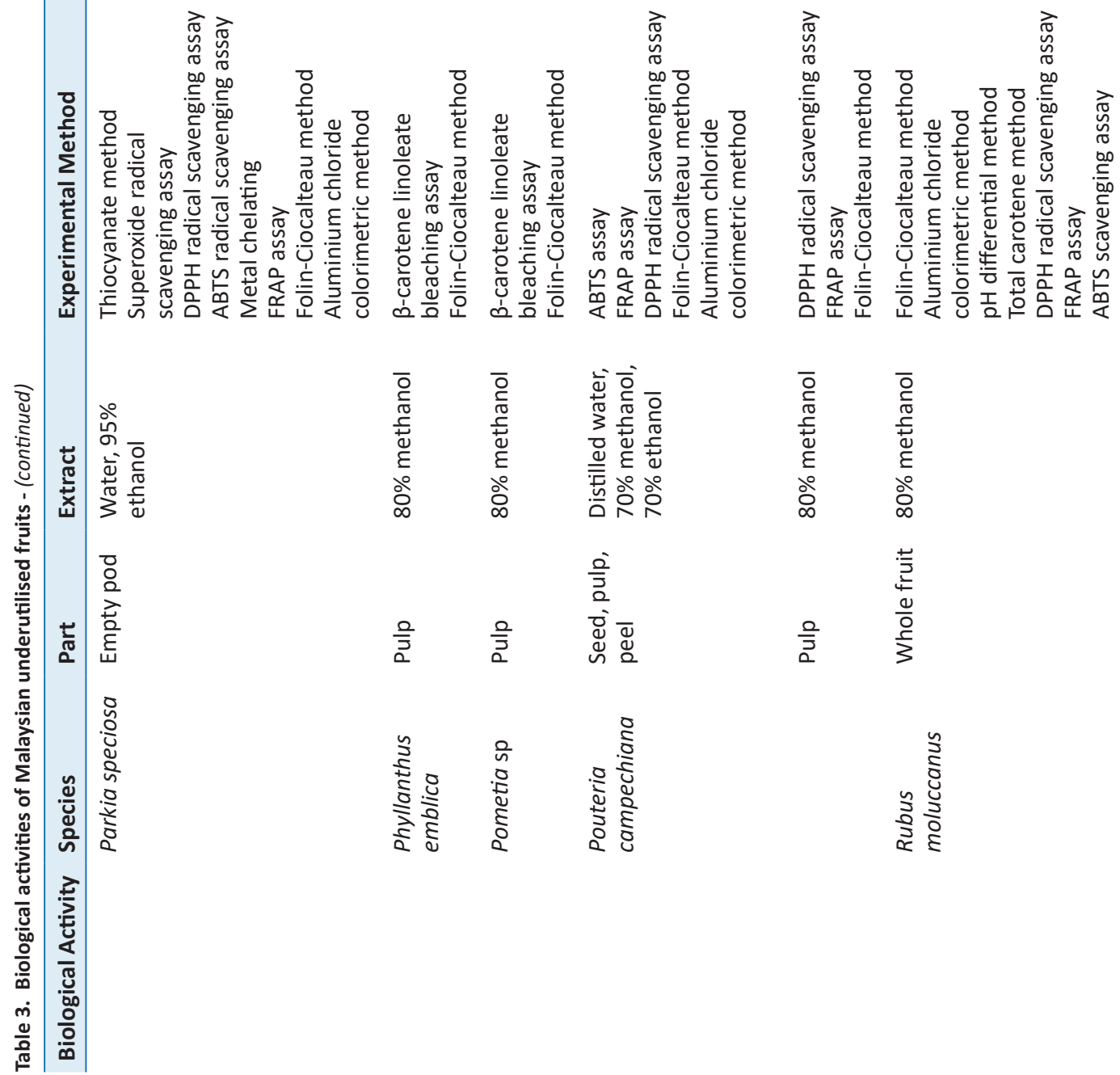

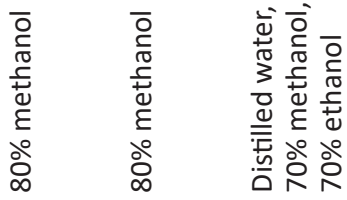

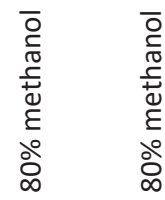

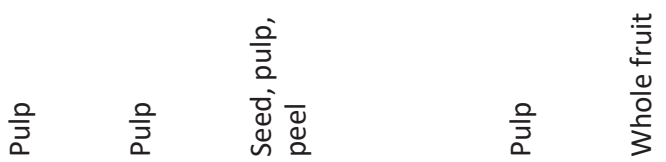

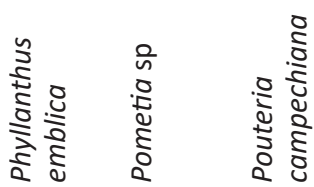

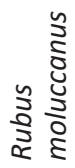

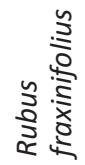




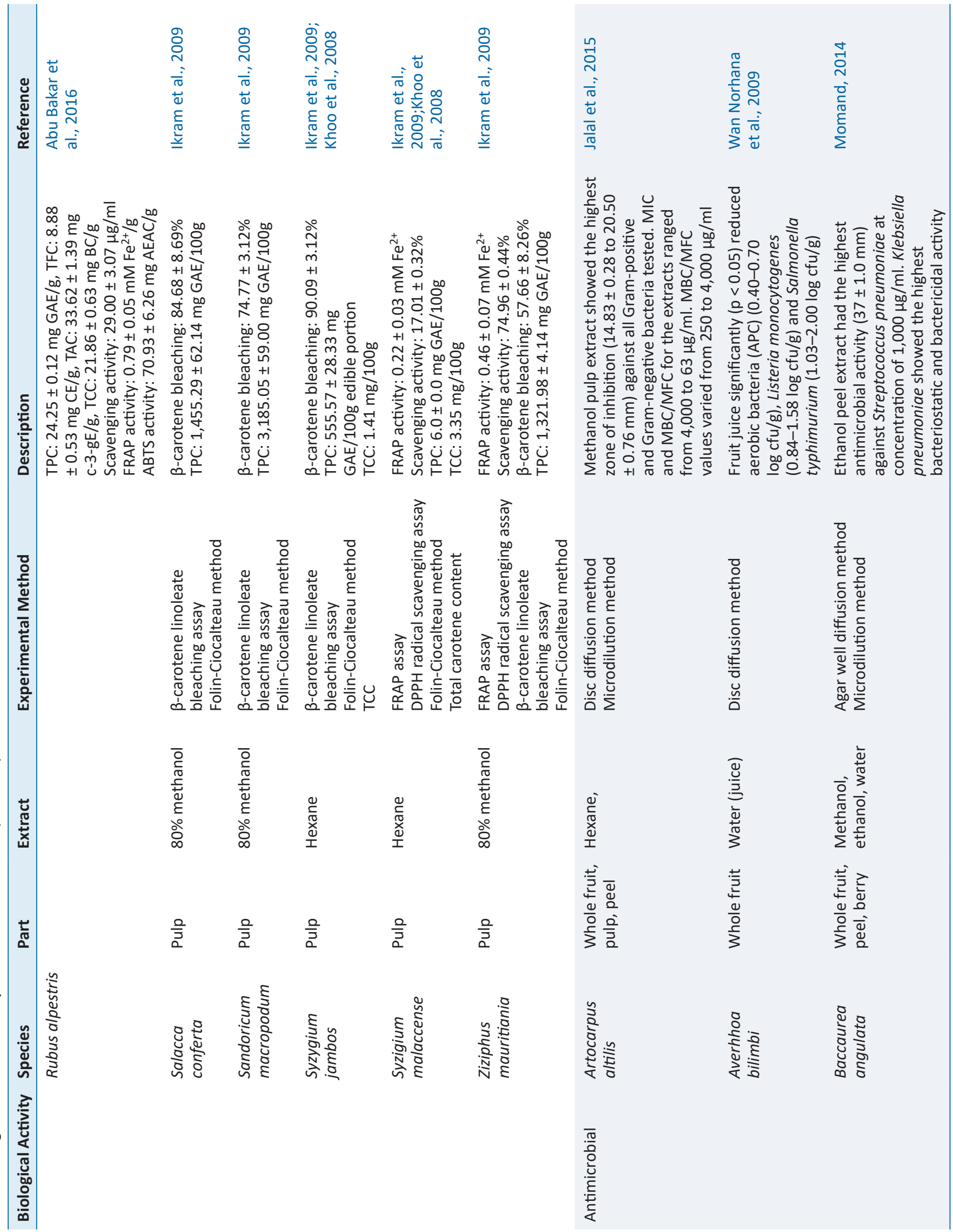




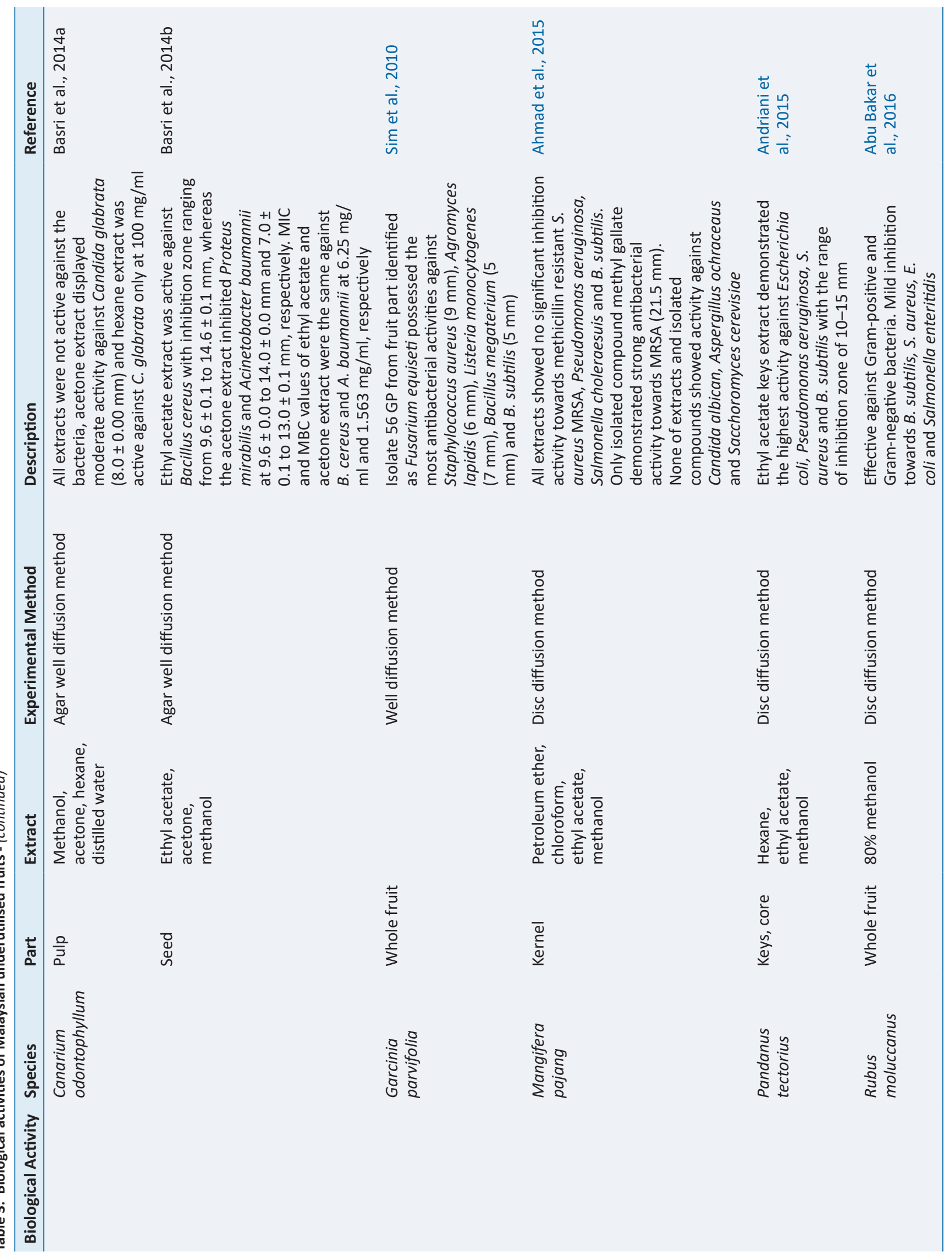









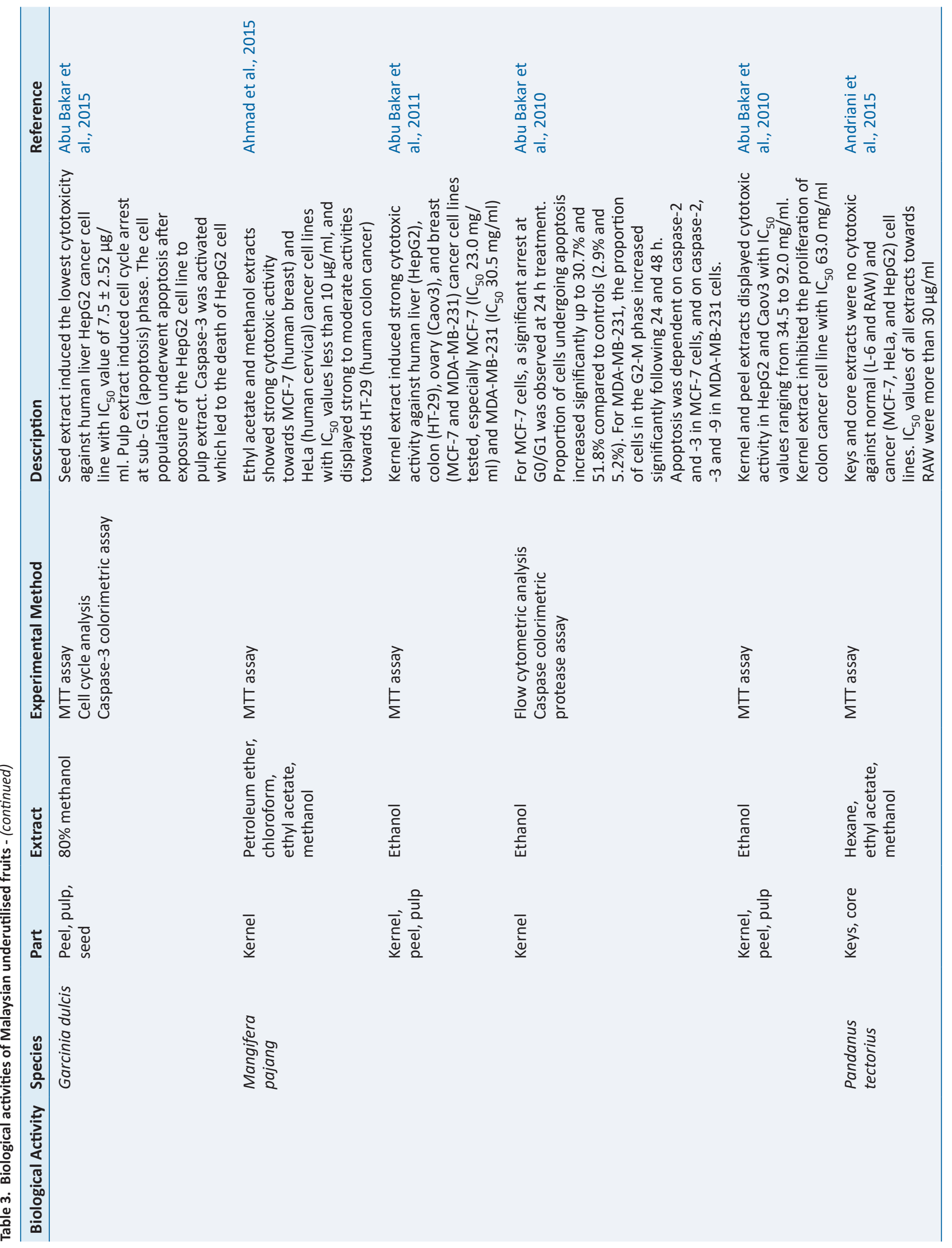




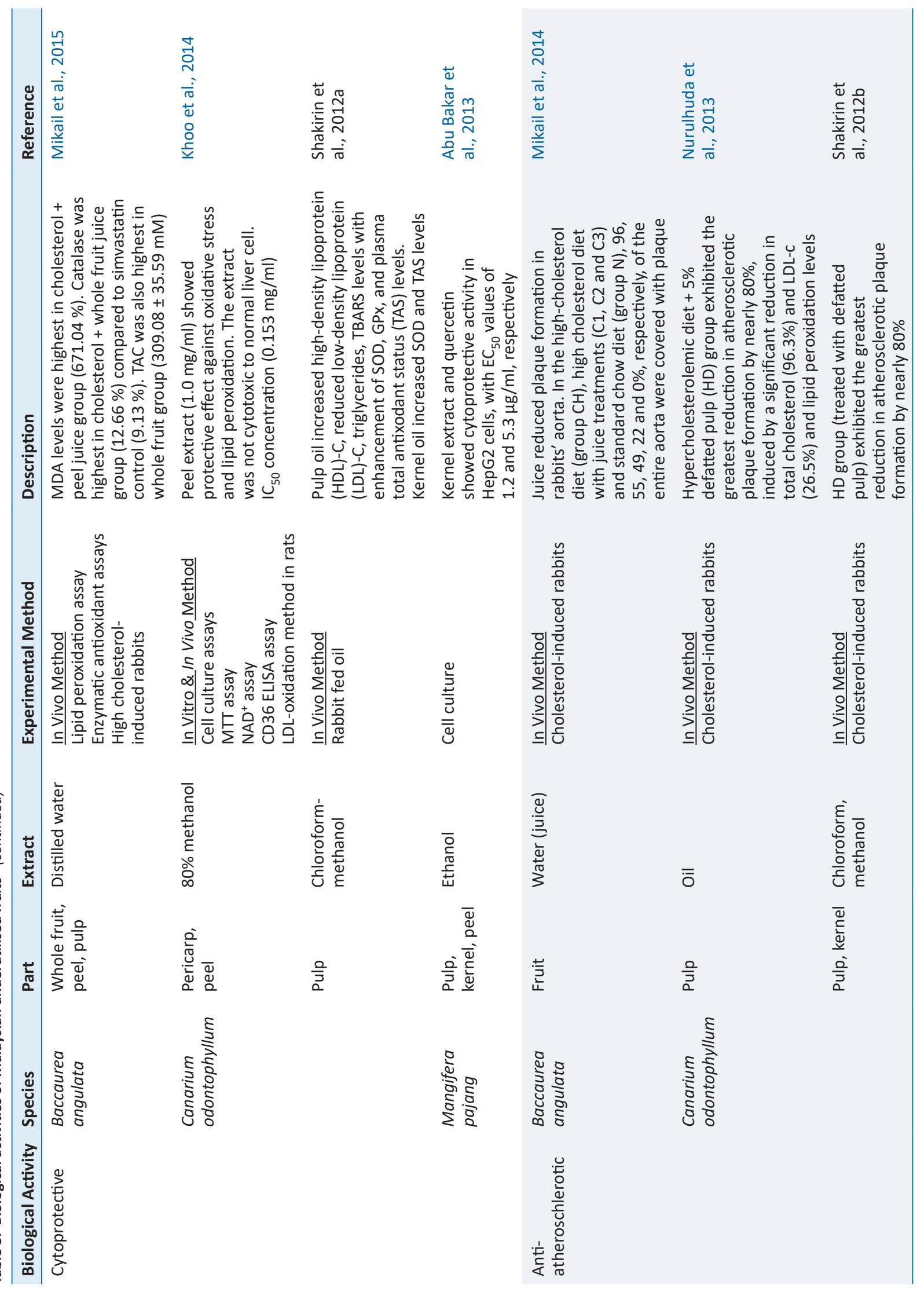




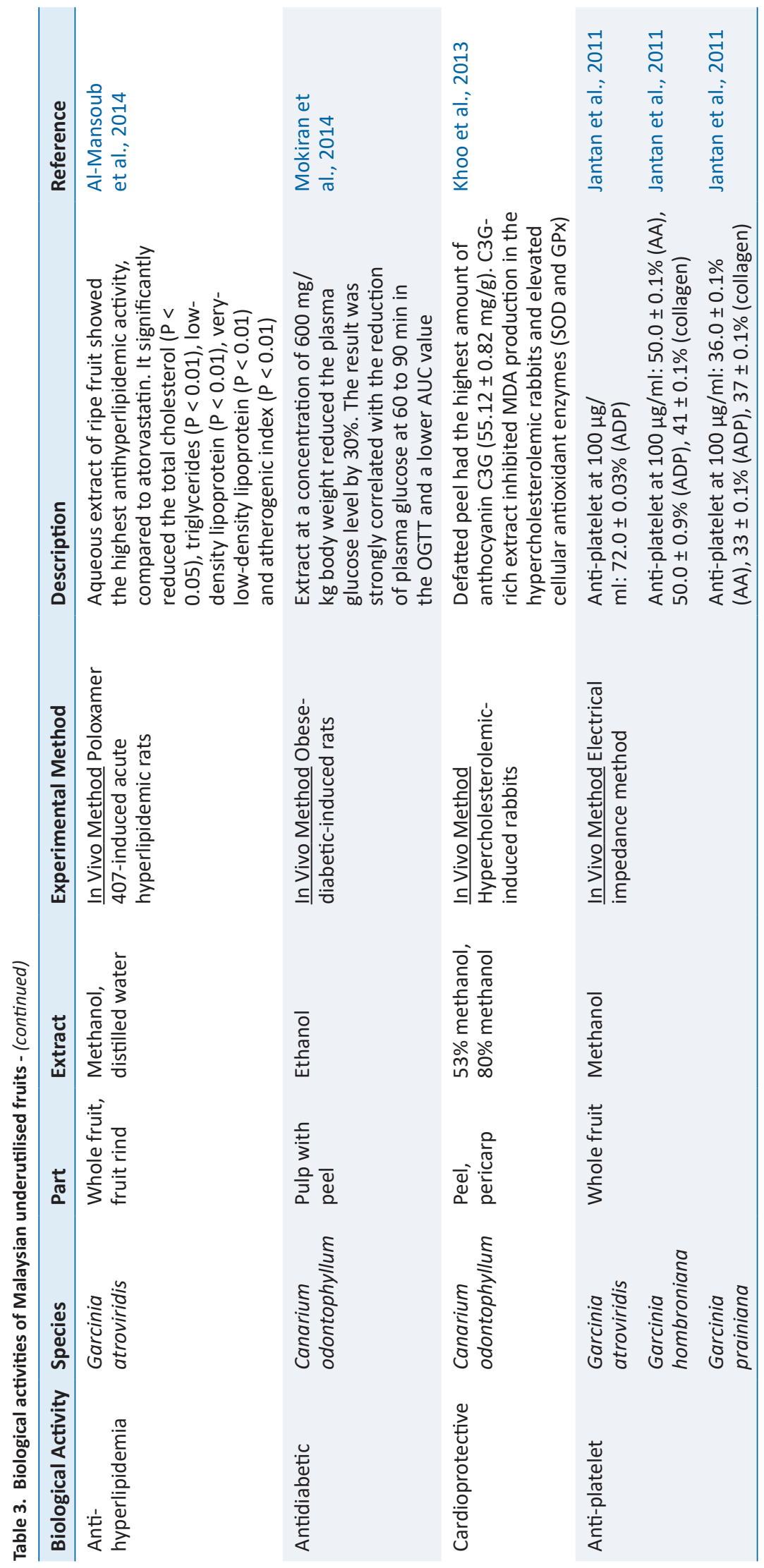




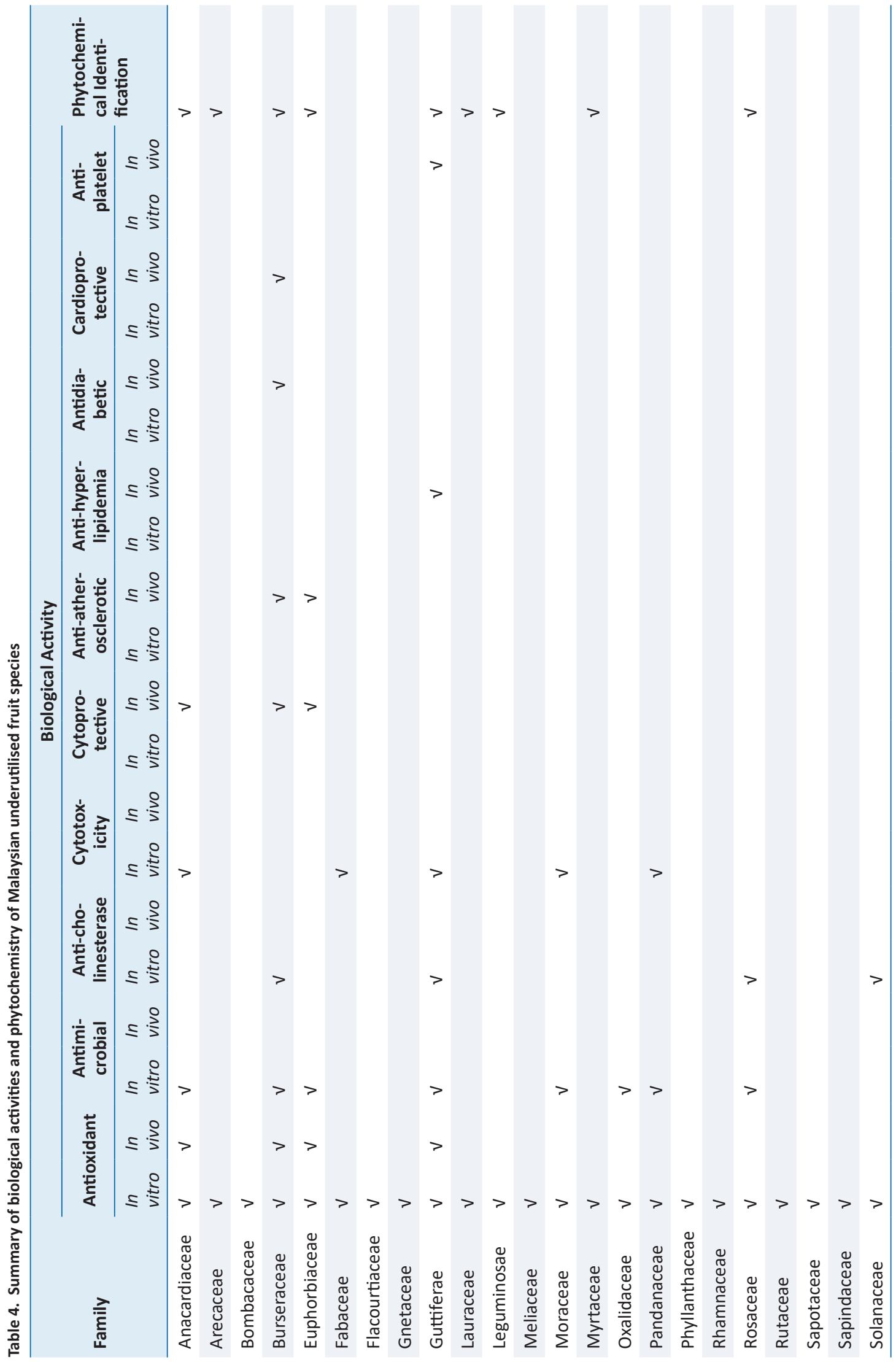


activity $(74.6 \pm 0.4 \%)$ than DPPH scavenging activity. Ali Hassan et al. (2013b) found that pulp extracts showed higher DPPH radical scavenging activity $(88.14 \pm 1.42 \%)$ and FRAP activity $(30.52 \pm$ $\left.0.54 \mathrm{mM} \mathrm{Fe}{ }^{2+} / 1\right)$. However, the FRAP activity of peel extracts as reported by Shakirin et al. (2010) was remarkably higher (1744 \pm $0.32 \mathrm{mM} \mathrm{Fe}{ }^{2+} / \mathrm{g}$ ). In in vivo study, it was observed that both peel and pericarp extracts of $C$. odontophyllum had significantly reduced lipid peroxidation effect as compared to the anthocyanin standard, cyanidin-3-glucoside. Thiobarbiturate reactive substance (TBARS) values among defatted peel, pericarp and cyanidin-3- glucoside showed no significant differences for hemoglobin and low density lipoprotein (LDL) oxidation assays (Khoo et al, 2014).

Meanwhile, among the abundant species of Anarcadiaceae family which have been tested for antioxidant activities, $M$. pajang or bambangan was the centre of attention. The findings of pulp (Abu Bakar et al., 2009; Abu Bakar et al., 2011; Ikram et al., 2009; Mirfat et al., 2015), peel (Abu Bakar et al., 2009; 2011; Ahmad et al., 2015; Hassan et al., 2011) and kernel (Abu Bakar et al., 2009; 2011; Ibrahim et al., 2013) were reported by six different research groups. Abu Bakar et al. (2009; 2011) described that methanolic kernel extract displayed the highest DPPH scavenging effect (23.23 $\mathrm{mg}$ ascorbic acid equivalent capacity $(\mathrm{AEAC}) / \mathrm{g}$ ) and FRAP reducing activity $\left(3,130.00 \pm 35.47 \mu \mathrm{M} \mathrm{Fe}^{2+} / \mathrm{g}\right)$. Meanwhile, other researchers reported that ethyl acetate and methanolic kernel extracts were strong DPPH radical scavengers with $\mathrm{IC}_{50}$ values of $7.28 \pm 0.30$ and $8.84 \pm 1.04 \mu \mathrm{g} / \mathrm{ml}$ (Ibrahim et al., 2013). Pulp was tested for $\beta$-carotene bleaching activity which ranged from $68.47 \pm 1.5648 .65 \pm 2.70 \%$ (Ikram et al., 2009) and DPPH radical scavenging activity with $\mathrm{IC}_{50}$ of $37.94 \pm 1.29 \mathrm{mg} / \mathrm{ml}$ (Mirfat et al., 2015). Peel was the only fruit part that was reported for in vivo study in human subjects. After administration of the peel juice extract, plasma total antioxidant status, plasma $\beta$-carotene and ascorbic acid were increased by 18,45 and $28 \%$ as compared to the baseline level and placebo, but liver and kidney functions were unaffected (Ahmad et al., 2015).

Both in vitro and in vivo assays were also performed in Baccaurea angulate, known as belimbing dayak. Based on the literature, four studies investigated the same whole fruit, pulp and peel parts (Ahmed et al., 2013; Ahmed et al., 2015; Jauhari et al., 2013; Mikail et al., 2014; 2015;). All researchers reported the same results that methanolic peel extracts displayed the highest DPPH radical scavenging activity with $96.80 \pm 0.53 \%$ (Ahmed et al., 2013; 2015 ) and $78.54 \pm 2.08 \mathrm{mg} \mathrm{AA} / 100 \mathrm{~g}$ (Jauhari et al., 2013). Jauhari et al. (2013) also reported that methanolic peel extract showed the highest FRAP activity $(50.86 \pm 4.24 \mathrm{~mm} \mathrm{TE} / \mathrm{g})$ and TEAC activity $(492.79 \pm 53.77 \mathrm{~mm}$ TE/100g). Mikail et al. (2015) studied the in vivo antioxidant effect of $B$. angulata fruits in rabbits. Plasma malondialdehyde (MDA) levels in high cholesterol-induce rabbits were the greatest in peel juice treated group (671.04\%), while whole fruit juice group demonstrated the highest level of catalase activity $(12.66 \%)$ and total antioxidant capacity $(309.08 \pm 35.59$ mM) (Mikail et al., 2015).

\subsection{Antimicrobial effect}

Ten species of underutilised fruits in Malaysia have been recorded for their antimicrobial activities which comprise A. altilis, A. bilimbi, B. angulata, C. odontophyllum, G. parvifolia, M. pajang, P. tectorius, R. moluccanus, R. fraxinifolius and R. alpestris (Table 3). Similar to antioxidant activities, these fruit species also exhibited varying degrees of antimicrobial activity depending on the fruit part, methods employed and extraction solvents used. The methods that were reported to assess antimicrobial activities were in the order of disc diffusion $>$ well diffusion $>$ microdilution. Few of the fruit species were further evaluated for minimal inhibitory concentration (MIC) and minimum bactericidal concentration (MBC)/ minimum fungicidal concentration (MFC). Several polar and nonpolar extraction solvents were used which was again dominated by methanol, and the extracts were tested against common pathogenic microbes; bacteria, fungi and yeasts.

Canarium odontophyllum fruits, again, were the main focus of the researchers. This is evident by the studies reported by Basri et al. (2014a; 2014b). They found that methanol, acetone, hexane and distilled water extracts of pulp were not active against all the Gram-negative and Gram-positive bacteria tested at concentrations of $25-100 \mathrm{mg} / \mathrm{ml}$. However, acetone extract displayed moderate activity against Candida glabrata $(8.0 \pm 0.00 \mathrm{~mm})$ and hexane extract was active against C. glabrata only at $100 \mathrm{mg} / \mathrm{ml}$ (Basri et al., 2014a). With regard to C. odontophyllum seed, Basri et al. (2014b) demonstrated that ethyl acetate extract was active against B. cereus $(9.6 \pm 0.1 \mathrm{~mm}$ to $14.6 \pm 0.1 \mathrm{~mm})$, whereas the acetone extract was capable of inhibiting Proteus mirabilis and Acinetobacter baumannii at $9.6 \pm 0.0$ to $14.0 \pm 0.0 \mathrm{~mm}$ and $7.0 \pm 0.1$ to $13.0 \pm 0.1 \mathrm{~mm}$, respectively. MIC and MBC values of ethyl acetate and acetone extract were the same against Bacillus cereus and $\mathrm{A}$. baumannii at 6.25 and $1.563 \mathrm{mg} / \mathrm{ml}$, respectively.

However, many other researchers claim stronger inhibitory activities against the tested microbes. For instance, Momand (2014) revealed that ethanolic extract of $\mathrm{B}$. angulata peel was an effective inhibitor $(37 \pm 1.0 \mathrm{~mm})$ against $\mathrm{S}$. pneumoniae at a concentration of $1,000 \mu \mathrm{g} / \mathrm{ml}$. Methanolic extract of A. altilis (sukun) pulp exhibited pronounced activity with inhibition zones ranging from 14.83 \pm 0.28 to $20.50 \pm 0.76 \mathrm{~mm}$ against all Gram-positive and Gramnegative bacteria tested (Jalal et al., 2015). Meanwhile, only isolated compound methyl gallate of M. pajang demonstrated strong antibacterial effect towards methicillin resistant Staphylococcus aureus (MRSA) (21.5 mm) (Ahmad et al., 2015). There was one study that isolated bacteria from G. parvifolia (kundong) fruits which was identified as Fusarium equiseti. The isolate (56 GP) possessed the most antibacterial activity against $\mathrm{S}$. aureus $(9 \mathrm{~mm})$, Agromyces lapidis $(6 \mathrm{~mm})$, Listeria monocytogenes $(7 \mathrm{~mm})$, Bacillus megaterium $(5 \mathrm{~mm})$ and B. subtilis $(5 \mathrm{~mm})$ (Sim et al., 2010).

\subsection{Anti-cholinesterase effect}

Alzheimer's disease is a form of dementia characterized by loss of central cholinergic neurons associated with a marked reduction in content of acetylcholinesterase (AChE), the enzyme responsible for the termination of nerve impulse transmission at cholinergic synapses (Ali Hassan et al., 2013b). Therefore, anticholinesterase activities that have been documented for Malaysian underutilised fruits serve as an important information for further possibilities to develop anticholinesterase drugs as one of the important approaches in the management of Alzheimer's disease. Six underutilised fruit species which consists of $C$. odontophyllum, C. betacea and G. parvifolia, R. moluccanus, $R$. fraxinifolius and $R$. alpestris have been investigated for their anticholinesterase activities (Table 3 ).

Ali Hassan et al. (2013b) discovered that both edible (pulp with peel) and inedible (seed) extracts of $C$. odontophyllum showed anticholinesterase activity when tested at $0-100 \mu \mathrm{g} / \mathrm{ml}$. However, the highest activity was seen in the seed (22.4\%). In addition, Ali Hassan and Abu Bakar (2013) also suggested that Cyphomandra betacea (buah cinta) extracts displayed anticholinesterase activity at higher concentrations $(50-250 \mu \mathrm{g} / \mathrm{ml})$, with the highest activity in the peel. Eighty percent methanolic extracts of G. parvifolia also exhibited anticholinesterase activity at the same concentrations of 
C. betacea. In particular, the highest activity of G. parvifolia was observed in the pulp at $14.3 \%$. However, the activity was much lower than that of the positive control, galanthamine (Ali Hassan et al., 2013c). A recent study by Abu Bakar et al. (2016) revealed that all methanolic extracts of $R$. moluccanus, $R$. alpestris and $R$. fraxinifolius had weak anticholinesterase activities with $26.42 \pm 1.41$, $25.30 \pm 1.56$ and $23.06 \pm 1.12 \%$, respectively, even when tested at the highest concentration $(5 \mathrm{mg} / \mathrm{ml})$. This is in comparison with Donepenzil (positive control) which showed complete inhibition activity $(100 \%)$ when tested at $1 \mathrm{mg} / \mathrm{ml}$.

\subsection{Cytotoxicity effect}

Five species of underutilised fruits in Malaysia have been studied for cytotoxicity; these include A. odoratissimus, C. cauliflora, G. dulcis, P. tectorius and the popular, M. pajang (Table 3). MTT ([3-(4,5-dimethylthiazol-2-yl)-2,5-diphenyltetrazolium bromide]) assay is commonly used by researchers to investigate the cytotoxicity of the fruit species. Out of the underutilised species, only $A$. odoratissimus was not cytotoxic towards all the cancer cell lines tested; human liver (HepG2), human colon (HT-29) and human ovarian (Caov3) (Abu Bakar et al. 2010).

Cynometra cauliflora, locally known as nam-nam, has traditionally been used in folk medicine (Tajudin et al., 2012); it exhibited a very strong cytotoxic activity towards human promyelocytic leukemia HL-60 cells $\left(\mathrm{CD}_{50} 0.9 \mu \mathrm{g} / \mathrm{ml}\right)$ and inhibited the cells into apoptotic cell death mode. However, it was less cytotoxic towards normal mouse fibroblast cell line 3T3/NIH cells. The authors stated that extracts which demonstrated $\mathrm{CD}_{50}$ value of $10-25 \mu \mathrm{g} / \mathrm{ml}$ were considered to be weak in cytotoxicity, while extracts with $\mathrm{CD}_{50}$ value of less than $5.0 \mu \mathrm{g} / \mathrm{ml}$ were considered very active (Tajudin et al., 2012). Another strong cytotoxic effect was demonstrated by G. dulcis (mundu) from the Guttiferae family (Abu Bakar et al., 2015). The seed extract induced the lowest cytotoxicity against HepG2 cancer cell line with $\mathrm{IC}_{50}$ value of $7.5 \pm 2.52 \mu \mathrm{g} / \mathrm{ml}$. Meanwhile, pulp extract induced cell cycle arrest at sub-G1 (apoptosis) phase in a time-dependent manner. The cell population underwent apoptosis after 72 hours of exposure of the HepG2 cell line to pulp extract. Caspase-3 was activated which led to the death of HepG2 cell (Abu Bakar et al., 2015). Another study revealed that ethyl acetate and methanol extracts from $M$. pajang kernel showed strong cytotoxic activity towards human breast (MCF-7) and cervical (HeLa) cancer cell lines (Ahmad et al., 2015). The remarkable effect was demonstrated in a dose-dependent manner with $\mathrm{IC}_{50}$ value of less than $10 \mu \mathrm{g} / \mathrm{ml}$. The extracts also displayed strong to moderate activities towards HT-29. This recent study was in agreement with the earlier study conducted by Abu Bakar et al. (2011). They reported that $M$. pajang methanolic kernel extract induced strong cytotoxic activities against HT-29, HepG2, Caov3, and especially breast (MCF-7 and MDA-MB-231) cancer cell lines tested. However, the $\mathrm{IC}_{50}$ of MCF-7 and MDA-MB-231 were much higher than $10 \mu \mathrm{g} / \mathrm{ml}$ with 23.0 and $30.5 \mathrm{mg} / \mathrm{ml}$, respectively. In a previous study by Abu Bakar et al. (2010), M. pajang ethanolic kernel extract was found to arrest the growth of proliferating cells from MCF-7 and MDA-MB-231 cancer cell lines, which was associated with induction of apoptosis as measured by cell cycle profiling, extrusion of phosphatidylserine to the outer surface of the plasma membrane, and induction of caspase activity.

\subsection{Cytoprotective effect}

Cytoprotective is strongly correlated with antioxidant as antioxi- dants protect biological structures from oxidative damage caused by free radicals. Of the all underutilised fruit species identified, $B$. angulata, C. odontophyllum and M. pajang were reported for their cytoprotective activity using in vitro and in vivo systems (Table 3 ).

Mikail et al. (2015) investigated the effects of $B$. angulata juice on plasma MDA levels, as well as the activities of antioxidant enzymes and total antioxidant capacity in rabbits. They showed that $B$. angulata fruit significantly $(\mathrm{p}<0.05)$ decreased the plasma level of MDA and increased the activities of superoxide dismutase (SOD), glutathione peroxidase (GPx) and catalase (CAT) in rabbits fed a high-cholesterol diet. The ability of $B$. angulata to protect low-density lipoprotein (LDL) from oxidative modification may be attributed to phenolic compounds known to act as powerful chain-breaking antioxidants and free radical scavengers. In another in vivo study, Shakirin et al. (2012a) suggested that the supplementation of pulp and kernel oils of $C$. odontophyllum in healthy rabbits might offer some protective effects against generation of free radicals, thus reducing the oxidative stress by enhancing the conversion of superoxide radicals to hydrogen peroxide $\left(\mathrm{H}_{2} \mathrm{O}_{2}\right)$, followed by deactivation of $\mathrm{H}_{2} \mathrm{O}_{2}$ by GPx. Meanwhile, in vitro cytoprotective study of pericarp and peel extracts of $C$. odontophyllum was evaluated using Chang liver cells (human normal) and HUVEC (human umbilical vein endothelial) cell lines (Khoo et al., 2014). Both extracts were found to exhibit cytoprotective effects in tert-butyl hydroperoxide (t-BHP) and 40\% methanol-induced cell death. They also showed no toxic effect to Chang liver cell line. However, using CD36 ELISA, NAD+ and LDL inhibition assays, inhibition of oxidative stress was found to be higher in the peel extract. $\mathrm{IC}_{50}$ concentration of the peel extract was greatly lower than the pericarp $(0.153 \mathrm{mg} / \mathrm{ml})$ indicating that peel extract had a stronger protective effect against oxidative damage compared to the pericarp extract. Mangifera pajang was also investigated for its potential in the protection against oxidative damage. Abu Bakar et al. (2013) reported that among pulp, kernel and peel extracts of M. pajang, kernel extract protected HepG2 cells against t-BHP induced-cell death. The extract gave a higher cytoprotection index than quercetin, with an $\mathrm{EC}_{50}$ value of $1.21 \pm 0.13 \mu \mathrm{g} / \mathrm{ml}$.

\subsection{Anti-atherosclerotic effect}

Although many species of underutilised fruits have been discovered in Malaysia, only B. angulata and C. odontophyllum fruit species were studied for their antiatherosclerotic properties (Table 3). Atherosclerosis is characterized by the accumulation of cholesterol deposits in the macrophages of arteries which if uncontrolled, may result in coronary artery heart disease (Shakirin et al., 2012b).

Baccaurea angulata was found to reduce atherosclerosis progression (Mikail et al., 2014). They described that the supplementation of $B$. angulata juice showed plaque-reducing activity in cholesterol-induced rabbits in a dose-dependent manner. In the high-cholesterol diet, high cholesterol diet with juice treatments $(0.5,1$ and $1.5 \mathrm{ml} / \mathrm{kg})$ and standard chow diet, plaque formation at the entire aorta were $96,55,49,22$ and $0 \%$, respectively. In another study, Nurulhuda et al. (2013) reported that high cholesterol animal group supplemented with defatted pulp powder exhibited the greatest reduction in atherosclerotic plaque formation by $79.4 \%$, induced by a significant reduction in total cholesterol $(96.3 \%)$ and LDL-c (26.5\%) and lipid peroxidation levels.

\subsection{Anti-hyperlipidemia effect}

Hyperlipidemia is a metabolic condition that can be due to an 
increase in blood lipid levels, which include cholesterol and triglycerides (Al-Mansoub et al., 2014). Only one antihyperlipidemia study was recorded from Malaysian underutilised fruit, G. atroviridis or locally known as asam gelugor (Table 3). This fruit species has demonstrated notable ethnomedicinal properties as reported by Gerten et al. (2015), Al-Mansoub et al. (2014) and Salma et al. (2006) (Table 1). With respect to scientific research, Al-Mansoub et al. (2014) demonstrated that aqueous extract of the ripe fruit showed the most potent antihyperlipidemic activity, comparable to that of commercial drug, atorvastatin. It also significantly reduced the total cholesterol, triglycerides, low-density lipoprotein, verylow-density lipoprotein and atherogenic index at $\mathrm{P}<0.01$.

\subsection{Antidiabetic effect}

Canarium odontophyllum has been screened by researchers to see their hyperglycemic effect against obese-diabetic-induced rats (Table 3). Mokiran et al. (2014) investigated the potential of the ethanolic pulp extracts in two different concentrations. They found that after a 4-week treatment period, the administration of the higher extract concentration $(600 \mathrm{mg} \mathrm{DE} / \mathrm{kg} \mathrm{bw})$ reduced the plasma glucose level by $30 \%$, but this reduction was not statistically significant. However, the result was strongly correlated with the reduction of plasma glucose at 60 to $90 \mathrm{~min}$ in the oral glucose tolerance test (OGTT) and a lower area under the curve (AUC) value. The high concentration extracts also failed to increase the insulin level but increased its sensitivity and reduced insulin resistance (HOMA-IR) only at the end of the experiment. This was the only study of antidiabetic effect ever reported of Malaysian underutilised fruits.

\subsection{Cardioprotective effect}

The cardioprotective effect of $C$. odontophyllum was conducted against hypercholesterolemic-induced rabbits using lipid peroxidation marker (plasma MDA) and antioxidant enzymes (SOD and GPx) as biomarkers (Khoo et al., 2013) (Table 3). These markers are useful in provision of information and confirmation of inhibition of oxidative stress and cardioprotective effect. It was reported that the methanolic defatted peel extract had the highest amount of anthocyanin, cyanidin-3-glucoside (C3G) with $55.12 \pm 0.82 \mathrm{mg} / \mathrm{g}$. This $\mathrm{C} 3 \mathrm{G}$-rich extract inhibited MDA production in the hypercholesterolemic rabbits and elevated cellular antioxidant enzymes (SOD and GPx). In particular, a significantly low increment (0.19 $\mathrm{mol} / \mathrm{l}$ ) of plasma MDA was observed for the treatment group after supplementation of $\mathrm{C} 3 \mathrm{G}$-rich extracts for 8 weeks as compared to control group. However, no significant change of GPx activity was found.

\subsection{Anti-platelet activity effect}

The study of the antiplatelet activity of Malaysian underutilised fruits was reported from Garcinia spp which comprised G. atroviridis, G. hombroniana and G. prainiana (Table 3). Platelets have been implicated in the pathogenesis of artherothrombotic conditions and play a key role in acute arterial thrombosis. Platelet aggregation was measured using electrical impedance method to assess the action of endogenous agonists such as arachidonic acid (AA), adenosine diphosphate (ADP), platelet activating factor (PAF), thrombin and collagen. Jantan et al. (2011) indicated that methanolic whole fruit extracts of G. atroviridis had pronounced inhibition of platelet in human subjects at $100 \mu \mathrm{g} / \mathrm{ml}$ with $72.0 \pm$ $0.03 \%$ (ADP). Garcinia hombroniana and G. prainiana demonstrated anti-platelet activities of $50.0 \pm 0.1 \%$ (AA), $50.0 \pm 0.9 \%$ (ADP), $41 \pm 0.1 \%$ (collagen) and $36.0 \pm 0.1 \%$ (AA), $33 \pm 0.1 \%$ (ADP), $37 \pm 0.1 \%$ (collagen), respectively. The study was performed in human subjects.

\section{Conclusion}

Underutilised fruits grown in tropical countries like Malaysia are not only an important source of food and nutrition for the rural population, but some of them have also been used in traditional medicine. This information is the basis to lead to a wide range of scientific studies. With regard to phytochemistry, only 21 underutilised fruit species have had their compounds identified and quantified. Phytochemical investigations of various parts of the fruits have revealed the presence of over 100 phytochemicals which comprise phenolics, terpenoids, carotenoids and other miscellaneous compounds. Meanwhile, about 51 underutilised fruit species have been identified and explored for various health promoting properties. Different parts of the fruits have been analysed mainly in vitro and barely in vivo. Research have shown that these underutilised fruits exhibit varying degrees of antioxidant, antimicrobial, anticholinesterase, cytotoxicity, antiatherosclerotic, antihyperlipidemia, antidiabetic, cytoprotective, cardioprotective and antiplatelet activity, although most of the studies are still not thorough. In addition, some species that have been used traditionally to treat many types of ailments have not been comprehensively investigated for their biological activities. Several fruit species for example, Mangifera species and Canarium odontophyllum have stolen the limelight that the remainder have been neglected by researchers. In a nutshell, this review has revealed that they are still gaps and inadequate information in some areas that open interesting doors for further research opportunities. More studies are needed to confirm the health significance and explain their mechanisms of action in order to fully understand the real potential of these underutilised fruit species.

\section{Acknowledgments}

Authors are grateful to Department of Nutrition and Dietetics, Faculty of Medicine and Health Sciences, University Putra Malaysia for financially supporting this work (GP-IPS/2017/9527300) and Malaysian Agricultural Research and Development Institute for their cooperation.

\section{References}

Abd Aziz, A.F., and Mohammad, I. (2013). Antioxidant activity and phytochemical composition of Cynometra cauliflora. J. Exp. Integr. Med. 3(4): 337-341.

Abd Ghafar, M.F., Prasad, K.N., Weng, K.K., and Ismail, A. (2010). Flavonoid, hesperidine, total phenolic contents and antioxidant activities from Citrus species. African J. Biotechnol. 9: 326-330.

Abraham, C.M. (2016). A study on phytochemical constituents of Averrhoa. Indian J. Appl. Res. 6: 29-31.

Abu Bakar, M.F., Ahmad, N., Karim, F., and Saib, S. (2014). Phytochemicals and antioxidative properties of Borneo indigenous liposu (Baccaurea lanceolata) and tampoi (Baccaurea macrocarpa) fruits. Antioxidants. 3: 516-525.

Abu Bakar, M.F., Ahmad, N.E., Suleiman, M., Rahmat, A., and Isha, A. 
(2015). Garcinia dulcis Fruit Extract induced cytotoxicity and apoptosis in HepG2 liver cancer cell line. BioMed Res. Int. 2015: 916902.

Abu Bakar, M.F., and Fry, J. (2013). A review on underutilized indigenous bambangan (Mangifera pajang) fruit as a potential novel source for functional food and medicine. J. Med. Plants Res. 7(45): 3292-3297.

Abu Bakar, M.F., Ismail, N.A., Isha, A., and Mei Ling, A.L. (2016). Phytochemical composition and biological activities of selected wild berries (Rubus moluccanus L., R. fraxinifolius Poir. and R. alpestris Blume). J. Evid. Based Complement. Altern. Med. 2016: 2482930.

Abu Bakar, M.F., Mohamad, M., Rahmat, A., Burr, S.A., and Fry, J.R. (2010). Cytotoxicity, cell cycle arrest, and apoptosis in breast cancer cell lines exposed to an extract of the seed kernel of Mangifera pajang (bambangan). Food Chem. Toxicol. 48(6): 1688-1697.

Abu Bakar, M.F., Mohamed, M., Rahmat, A., Burr, S.A., and Fry, J.R. (2010). Cytotoxicity and polyphenol diversity in selected parts of Mangifera pajang and Artocarpus odoratissimus fruits. Nutr. Food Sci. 40(1): 29-38.

Abu Bakar, M.F., Mohamed, M., Rahmat, A., Burr, S.A., and Fry, J.R. (2013). Cellular assessment of the extract of bambangan (Mangifera pajang) as a potential cytoprotective agent for the human hepatocellular HepG2 cell line. Food Chem. 136(1): 18-25.

Abu Bakar, M.F., Mohamed, M., Rahmat, A., and Fry, J. (2009). Phytochemicals and antioxidant activity of different parts of bambangan (Mangifera pajang) and tarap (Artocarpus odoratissimus). Food Chem. 113(2): 479-483.

Abu Bakar, M.F., Mohamed, M., Rahmat, A., and Fry, J.R. (2011). Bambangan (Mangifera pajang) Seed Kernel: Antioxidant Properties and Anti-cancer Effects. Nuts and Seeds in Health and Disease Prevention 183-187.

Ahmad, S., Sukari, M.A., Ismail, N., Ismail, I.S., Abdul, A.B., Abu Bakar, M.F., and Ee, G.C.L. (2015). Phytochemicals from Mangifera pajang Kosterm and their biological activities. BMC Complement. Altern. Med. 15(1): 83

Ahmed, I.A., Maryam Abimbola, M., Muhammad, I., Norazlanshah, H., Mohammad Syaiful Bahari, A.R., Radiah, A.G., and Mohammad Nor Adros, Y. (2013). In vitro antioxidant properties of underutilized Baccaurea angulata fruit. Int. J. Adv. Agric. Environ. Eng. 1(1): 144-150.

Ahmed, I.A., Mikail, M.A., Ibrahim, M., Hazali, N., Rasad, M.S.B.A., Ghani, R.A., and Yahya, M.N.A. (2015). Antioxidant activity and phenolic profile of various morphological parts of underutilised Baccaurea angulata fruit. Food Chem. 172: 778-787.

Ali Hassan, S.H., and Abu Bakar, M.F. (2013). Antioxidative and anticholinesterase activity of Cyphomandra betacea fruit. Sci. World J. 2018 : 278071.

Ali Hassan, S.H., Fry, J.R., and Abu Bakar, M.F. (2013a). Antioxidant and phytochemical study on pengolaban (Litsea garciae), an edible underutilized fruit endemic to Borneo. Food Sci. Biotechnol. 22(5): 1-7.

Ali Hassan, S.H., Fry, J.R., and Abu Bakar, M.F. (2013b). Phytochemicals content, antioxidant activity and acetylcholinesterase inhibition properties of indigenous Garcinia parvifolia fruit. BioMed Res. Int. 2013: 138950

Ali Hassan, S.-H., Fry, J.R., and Abu Bakar, M.F. (2013). Antioxidative phytochemicals and anti-cholinesterase activity of native kembayau (Canarium odontophyllum) fruit of Sabah, Malaysian Borneo. J. Nutr. Food Sci. 4(1): 1-6.

Al-Mansoub, M.A., Asmawi, M.Z., and Murugaiyah, V. (2014). Effect of extraction solvents and plant parts used on the antihyperlipidemic and antioxidant effects of Garcinia atroviridis: A comparative study. J. Sci. Food Agric. 94(8): 1552-1558.

Andriani, Y., Ramli, N.M., Syamsumir, D.F., Kassim, M.N.I., Jaafar, J., Aziz, N.A., and Mohamad, H. (2015). Phytochemical analysis, antioxidant, antibacterial and cytotoxicity properties of keys and cores part of Pandanus tectorius fruits. Arab. J. Chem.

Anupunt, P., Somsri, S., Chaikiattiyos, S., and Kumcha, U. (2003). Native tropical Asian fruits. Acta Hort. 620: 151-159.

Azlan, A., Ismail, A., Ibrahim, M., Shakirin, F.H., and Khoo, H.E. (2013). Health-promoting properties of selected Malaysian underutilized fruits. Acta Hort. 979: 203-210.

Azlan, A., Prasad, K.N., Khoo, H.E., Abdul-Aziz, N., Mohamad, A., Ismail, A., and Amom, Z. (2010). Comparison of fatty acids, vitamin $E$ and physicochemical properties of Canarium odontophyllum Miq. (dabai), ol- ive and palm oils. J. Food Compost. Anal. 23(8): 772-776.

Basri, D.F., Fairuzishak, S., and Zin, N.M. (2014). Shell extract of seed from Canarium odontophyllum Miq. (dabai) fruit as potential source of antibacterial agent. Int. J. Pharm. Sci. Rev. Res. 28(2): 257-262.

Basri, D.F., Saidi, N., Mahari, H., Saari, S., and Santhanam, J. (2014). Preliminary screening for antimicrobial activity of the pulp of Canarium odontophyllum Miq. (dabai) fruit. Global J. Pharmacol. 8(2): 213-220.

Bhat, R., and Yahya, N. (2014). Evaluating belinjau (Gnetum gnemon L.) seed flour quality as a base for development of novel food products and food formulations. Food Chem. 156: 42-49.

Chau Sum, P., Eng Khoo, H., and Azlan, A. (2013). Comparison of nutrient composition of ripe and unripe fruits of Nypa fruticans. Fruits. 68(6): 491-498.

Cheong, M.W., Zhu, D., Sng, J., Liu, S.Q., Zhou, W., Curran, P., and Yu, B. (2012). Characterisation of calamansi (Citrus microcarpa). Part II: Volatiles, physicochemical properties and non-volatiles in the juice. Food Chem. 134(2): 696-703.

Chew, L.Y., Prasad, K.N., Amin, I., Azrina, A., and Lau, C.Y. (2011). Nutritional composition and antioxidant properties of Canarium odontophyllum Miq. (dabai) fruits. J. Food Compost. Anal. 24(4-5): 670-677.

Chew, Y.L., Chan, E.W.L., Tan, P.L., Lim, Y.Y., Stanslas, J., and Goh, J.K. (2011). Assessment of phytochemical content, polyphenolic composition, antioxidant and antibacterial activities of Leguminosae medicinal plants in Peninsular Malaysia. BMC Complement. Altern. Med. 2(1): 12.

Gerten, D., Salma, I., Shafie, M.S.M., Shariah, U., Brooke, P., Wong, W.W.W. and Norhayati, M.H. (2015). Traditional knowledge and practices related to genus Citrus, Garcinia, Mangifera and Nephelium in Malaysia. OALib. 2(4): 1-11.

Hassan, F.A., Ismail, A., Abdul Hamid, A., and Azlan, A. (2011). Identification and quantification of phenolic compounds in bambangan (Mangifera pajang kort.) Peels and their free radical scavenging activity. J. Agric. Food Chem. 59(17): 9102-9111.

Hassan, F.A., Ismail, A., Abdul Hamid, A., Azlan, A., and Al-Sheraji, S.H. (2011). Characterisation of fibre-rich powder and antioxidant capacity of Mangifera pajang K. fruit peels. Food Chem. 126(1): 283-288.

Husen, R. (2015). Potential use of superheated-steam treatment in underutilized fruit of engkala (Litsea garciae) and evaluation of its antioxidant capacity. Thesis of Kyushu Institute of Technology, Japan.

Ibrahim, M., Ismail, A., Al-Sheraji, S.H., Azlan, A., and Abdul Hamid, A. (2013). Effects of Mangifera pajang Kostermans juice on plasma antioxidant status and liver and kidney function in normocholesterolemic subjects. J. Funct. Food. 5(4): 1900-1908.

Ikram, E.H.K., Eng, K.H., Jalil, A.M.M., Ismail, A., Idris, S., Azlan, A., and Mokhtar, R.A.M. (2009). Antioxidant capacity and total phenolic content of Malaysian underutilized fruits. J. Food Compost. Anal. 22(5): 388-393.

Jalal, T.K., Ahmed, I.A., Mikail, M., Momand, L., Draman, S., Isa, M.L.M., and Abdul Wahab, R. (2015). Evaluation of antioxidant, total phenol and flavonoid content and antimicrobial activities of Artocarpus altilis (breadfruit) of underutilized tropical fruit extracts. Appl. Biochem. Biotechnol. 175(7): 3231-3243.

Jantan, I., Jumuddin, F.A., Saputri, F.C., and Rahman, K. (2011). Inhibitory effects of the extracts of Garcinia species on human low-density lipoprotein peroxidation and platelet aggregation in relation to their total phenolic contents. J. Med. Plants Res. 5(13): 2699-2709.

Jauhari, N.K., Ibrahim, D., Ibrahim, M., Yahya, M.N.A., Nor, N.M., Isa, K.A.M., and Hazali, N. (2013). Proximate composition and antioxidant activity of dried belimbing dayak (Baccaurea angulata) fruits. Sains Malays. 42(2): 129-134.

Khoo, H.E., Azlan, A., Ismail, A., and Abas, F. (2012a). Antioxidative properties of defatted dabai pulp and peel prepared by solid phase extrac tion. Molecules. 17(8): 9754-9773.

Khoo, H.E., Azlan, A., Ismail, A., and Abas, F. (2012b). Influence of different extraction media on phenolic contents and antioxidant capacity of defatted dabai (Canarium odontophyllum) fruit. Food Anal. Method. 5(3): 339-350.

Khoo, H.E., Azlan, A., Ismail, A., Abas, F., and Hamid, M. (2014). Inhibition of oxidative stress and lipid peroxidation by anthocyanins from defatted Canarium odontophyllum pericarp and peel using in vitro bioassays. PLOS ONE. 9(1): 1-9. 
Khoo, H.E., Azlan, A., Kong, K.W., and Ismail, A. (2016). Phytochemicals and medicinal properties of indigenous tropical fruits with potential for commercial development. J. Evid. Based Complement. Altern. 2016: 7591951.

Khoo, H.E., Azlan, A., Nurulhuda, M.H., Ismail, A., Abas, F., Hamid, M., and Roowi, S. (2013). Antioxidative and cardioprotective properties of anthocyanins from defatted dabai extracts. J. Evid. Based Complement. Altern. 2013: 434057

Khoo, H.E., Ismail, A., Mohd-Esa, N., and Idris, S. (2008). Carotenoid content of underutilized tropical fruits. Plant Food. Hum. Nutr. 63(4): 170-175.

Ko, H.-J., Ang, L.-H., and Ng, L.-T. (2014). Antioxidant activities and polyphenolic constituents of bitter bean Parkia speciosa. Int. J. Food Prop. 17(9): 1977-1986.

Kong, K.W., Chew, L.Y., Prasad, K.N., Lau, C.Y., Ismail, A., Sun, J., and Hosseinpoursarmadi, B. (2011). Nutritional constituents and antioxidant properties of indigenous kembayau (Dacryodes rostrata (Blume) H.J. Lam) fruits. Food Res. Int. 44(7): 2332-2338.

Kong, K.W., Khoo, H.E., Prasad, N.K., Chew, L.Y., and Amin, I. (2013). Total phenolics and antioxidant activities of Pouteria campechiana fruit parts. Sains Malays. 42(2): 123-127.

Kubola, J., Siriamornpun, S., and Meeso, N. (2011). Phytochemicals, vitamin C and sugar content of Thai wild fruits. Food Chem. 126(3): 972-981.

Mikail, M.A., Ahmed, I.A., Ibrahim, M., Hazali, N., Abdul Rasad, M.S.B., Abdul Ghani, R., and Adros Yahya, M.N. (2015). Baccaurea angulata fruit inhibits lipid peroxidation and induces the increase in antioxidant enzyme activities. Eur. J. Nutr. 55(4): 1435-1444.

Mikail, M.A., Ahmed, I.A., Ibrahim, M., Hazali, N., Abdul Rasad, M.S.B., Abdul Ghani, R., and Yahya, M.N.A. (2014). Changes in the markers of atherosclerosis following administration of belimbing dayak (Baccaurea angulata) fruit juice in experimental rabbits fed with cholesterol diet. Int. J. Adv. Agric. Environ. Eng. 1(1): 151-154.

Mirfat, A.H.S., Salma, I., and Razali, M. (2016). Natural antioxidant properties of selected wild Mangifera species in Malaysia. J. Trop. Agric. Food Sci. 44(1): 1-13.

Mirfat, A., Razali, M., and Salma, I. Umi Kalsum, H.Z. (2015). Antioxidant and nutritional values of selected underutilised Mangifera species in Malaysia. Indian J. Plant Gen. Resour. 28(1): 72-79.

Mohamed, A. (2012). Nutrient compostion and antioxidant properties of "kerekup" fruit (Flacourtia jangomas). Thesis of Universiti Teknologi Mara, Malaysia.

Mokhtar, S.I., Leong, P.C., Ven, L.E., and Nur Ain, A.A. (2014). Total phenolic contents, antioxidant activities and organic acids composition of three selected fruit extracts at different maturity stages. J. Trop. Resour. Sustain. Sci. 2: 40-46.

Mokiran, N.N., Ismail, A., Azlan, A., Hamid, M., and Hassan, F.A. (2014). Effect of dabai (Canarium odontophyllum) fruit extract on biochemical parameters of induced obese-diabetic rats. J. Funct. Food. 8(1): 139-149.

Momand, L. (2014). Antimicrobial effect of Baccaurea angulata fruit extracts against human pathogenic microorganisms. J. Med. Aromat. Plant. 3(4): 229-237.

Muhamad, N., Muhmed, S.A., Yusoff, M.M., and Gimbun, J. (2014). Influence of solvent polarity and conditions on extraction of antioxidant, flavonoids and phenolic content from Averrhoa bilimbi. J. Food Sci. Eng. 4: 255-260.

Noor, A., and Noriham, A. (2014). Antioxidant activity and bioactive components of Oxalidaceae fruit extracts. Malaysian J. Anal. Sci. 18(1): 116-126.

Nurulhuda, M.H., Azlan, A., Ismail, A., Amom, Z., and Shakirin, F.H. (2013). Sibu olive inhibits artherosclerosis by cholesterol lowering effect in cholesterol fed-rabbit. IFMBE Proc. 40: 141-144.

Prasad, K.N., Chew, L.Y., Khoo, H.E., Kong, K.W., Azlan, A., and Ismail, A.
(2010). Antioxidant capacities of peel, pulp, and seed fractions of Canarium odontophyllum Miq. fruit. J. Biomed. Biotechnol. 2019: 871379.

Prasad, K.N., Chew, L.Y., Khoo, H.E., Yang, B., Azlan, A., and Ismail, A. (2011). Carotenoids and antioxidant capacities from Canarium odontophyllum Miq. fruit. Food Chem. 124(4): 1549-1555.

Prasad, K.N., Hassan, F.A., Yang, B., Kong, K.W., Ramanan, R.N., Azlan, A., and Ismail, A. (2011). Response surface optimisation for the extraction of phenolic compounds and antioxidant capacities of underutilised Mangifera pajang Kosterm. peels. Food Chem. 128(4): 11211127.

Prasad, N., Yang, B., Kong, K.W., Khoo, H.E., Sun, J., Azlan, A., and Romli, Z. (2013). Phytochemicals and Antioxidant Capacity from Nypa fruticans Wurmb. Fruit. J. Evid. Based Complement. Altern. 2013: 154606.

Rajan, N.S., and Bhat, R. (2016). Volatile constituents of unripe and ripe kundang fruits (Bouea macrophylla Griffith). Int. J. Food Prop. 20: $1-22$.

Rajan, N.S., Bhat, R., and Karim, A.A. (2014). Preliminary studies on the evaluation of nutritional composition of unripe and ripe 'Kundang' fruits (Bouea macrophylla Griffith). Int. Food Res. J. 21(3): 949-954.

Salma, I., Masrom, H., and Raziah, M.L. (2006). Diversity and use of traditional fruit species in selected home gardens or fruit orchards in Malaysia. J. Trop. Agric. Food Sci. 34(1): 149-164.

Shakirin, F.H., Azlan, A., Ismail, A., Amom, Z., and Yuon, L.C. (2012). Protective effect of pulp oil extracted from Canarium odontophyllum Miq. fruit on blood lipids, lipid peroxidation, and antioxidant status in healthy rabbits. Oxid. Med. Cell. Longev. 2012: 840973.

Shakirin, F.H., Azlan, A., Ismail, A., Amom, Z., and Yuon, L.C. (2012). Antiatherosclerotic Effect of Canarium odontophyllum Miq. Fruit Parts in Rabbits Fed High Cholesterol Diet. J. Evid. Based Complement. Altern. Med. 2012: 838604.

Shakirin, F.H., Prasad, K.N., Ismail, A., Yuon, L.C., and Azlan, A. (2010). Antioxidant capacity of underutilized Malaysian Canarium odontophyllum (dabai) Miq. fruit. J. Food Compost. Anal. 23(8): 777-781.

Sim, J.H., Khoo, C.H., Lee, L.H., and Cheah, Y.K. (2010). Molecular diversity of fungal endophytes isolated from Garcinia mangostana and Garcinia parvifolia. J. Microbiol. Biotechnol. 20(4): 651-658.

Sulaiman, S.F., and Ooi, K.L. (2012). Polyphenolics, vitamin C, and antioxidant activities of aqueous extracts from mature-green and ripe fruit fleshes of Mangifera sp. J. Agric. Food Chem. 60: 1-25.

Tajudin, T.J.S.A., Mat, N., Siti-Aishah, A.B., Yusran, A.A.M., Alwi, A., and Ali, A.M. (2012). Cytotoxicity, antiproliferative effects, and apoptosis induction of methanolic extract of Cynometra cauliflora Linn. whole fruit on human promyelocytic leukemia HL-60 cells. J. Evid. Based Complement. Altern. Med. 2012: 127373.

Tee, L.H., Ramanan, R.N., Tey, B.T., Chan, E.S., Azrina, A., Amin, I., Yang, B., Lau, C.Y., and Prasad, K.N. (2015). Phytochemicals and antioxidant capacities from Dacryodes rostrata fruits. Med Chem. 5(1): 23-27.

Tee, L.H., Yang, B., Prasad, N.K., Ramanan, R.N., Sun, J., Chan, E.S., and Jiang, Y. (2014). Nutritional compositions and bioactivities of Dacryodes species: A review. Food Chem. 165: 247-255.

Voon, B.H., and Kueh, H.S. (1999). The nutritional value of indigenous fruits and vegetables in Sarawak. Asia Pac. J. Clin. Nutr. 8(1): 24-31.

Wan Norhana, M.N., A, Poole, S.E., Deeth, H.C., and Dykes, G.A. (2009). Effects of bilimbi (Averrhoa bilimbi L.) and tamarind (Tamarindus indica L.) juice on Listeria monocytogenes Scott A and Salmonella typhimurium ATCC 14028 and the sensory properties of raw shrimps. Int. J. Food Microbiol. 136(1): 88-94.

Wetwitayaklung, P., Charoenteeraboon, J., Limmatvapirat, C., and Phaechamud, T. (2012). Antioxidant activities of some Thai and exotic fruits cultivated in Thailand. Res. J. Pharm. Biol. Chem. Sci. 3(1): 12-21.

Wong, K.C., and Lai, F.Y. (1996). Volatile constituents from the fruits of four Syzygium species grown in Malaysia. Flavour Fragr. J. 11: 61-66. 